\title{
An Integrated Passive Phase-Shift Keying Modulator for Biomedical Implants With Power Telemetry Over a Single Inductive Link
}

\author{
Dai Jiang, Member, IEEE, Dominik Cirmirakis, Matthew Schormans, Student Member, IEEE, \\ Timothy A. Perkins, Nick Donaldson, and Andreas Demosthenous, Senior Member, IEEE
}

\begin{abstract}
This paper presents a passive phase-shift keying (PPSK) modulator for uplink data transmission for biomedical implants with simultaneous power and data transmission over a single 13.56 MHz inductive link. The PPSK modulator provides a data rate up to $1.35 \mathrm{Mbps}$ with a modulation index between $3 \%$ and $38 \%$ for a variation of the coupling coefficient between 0.05 and 0.26 . This modulation scheme is particularly suited for biomedical implants that have high power demand and low coupling coefficients. The PPSK modulator operates in conjunction with onoff-keying downlink communication. The same inductive link is used to provide up to $100 \mathrm{~mW}$ of power to a multi-channel stimulator. The majority of the system on the implant side was implemented as an application specific integrated circuit (ASIC), fabricated in 0.6- $\mu \mathrm{m}$ high voltage CMOS technology. The theory of PPSK modulation, simulated and measured performance evaluation, and comparison with other state-of-the-art impedance modulation techniques is presented. The measured bit error rate around critical coupling at $1.35 \mathrm{Mbps}$ is below $6 \times 10^{-8}$.
\end{abstract}

Index Terms-Application specific integrated circuit (ASIC), implantable device, inductive link, passive phase-shift keying (PPSK), power and data telemetry.

\section{INTRODUCTION}

$\mathbf{I}$ NDUCTIVELY powered implantable neural stimulators, such as cochlear implants [1], visual prostheses [2]-[5], spinal cord stimulators [6], [7], deep brain stimulators [8] and vestibular prostheses [9]-[12], are often equipped with back telemetry, over which the device operational parameters, such as electrode voltage, power supply level, humidity and temper-

Manuscript received November 25, 2015; revised March 15, 2016 and April 24, 2016; accepted May 12, 2016. This work was funded by the European Commission under Grant 225929 and the Engineering and Physical Sciences Research Council (EPSRC) under Grant EP/F009593/1. This paper was recommended by Associate Editor P. Mercier.

D. Jiang, M. Schormans, and A. Demosthenous are with the Department of Electronic and Electrical Engineering, University College London (UCL), London WC1E 7JE, U.K. (e-mail: d.jiang@ucl.ac.uk; matthew.schormans.10@ ucl.ac.uk; a.demosthenous@ucl.ac.uk).

D. Cirmirakis was with the Department of Electronic and Electrical Engineering, University College London (UCL), London WC1E 7JE, U.K. He is now with BlueCrest Capital Management (UK) LLP, London SW1X 7AW, U.K. (e-mail: Dominik.cirmirakis.09@alumni.ucl.ac.uk).

T. A. Perkins and N. Donaldson are with the UCL Department of Medical Physics and Bioengineering, London WC1E6BT, U.K. (e-mail: t.perkins@ucl. ac.uk; n.donaldson@ucl.ac.uk).

Color versions of one or more of the figures in this paper are available online at http://ieeexplore.ieee.org.

Digital Object Identifier 10.1109/TBCAS.2016.2580513 ature, are reported to external controllers for optimising power transfer and monitoring implant safety. In some applications, back telemetry is also used to record neural activities and transmit the digitised readings to an external processor, where the data can be analysed and integrated into neural modulation algorithms for closed-loop stimulation control [1], [12]-[14].

Transmission of multi-channel neural recording data from an inductively powered implant is technically challenging. On one hand, a wide bandwidth is preferable to allow high resolution real-time recording; on the other hand, the implementation must take account of the tight restriction on the physical size and power consumption. Active transmitters provide wide bandwidth at the cost of extra components such as RF antenna and power consumption [15]-[17]. The alternative of passive impedance modulation has been widely used because of its circuit simplicity and power efficiency [18]-[31]. However, a tradeoff exists in impedance modulation between the modulation index, data rate and power transfer efficiency. The coil size constraints for biomedical implants result in small coupling coefficients over the inductive link. In addition, some implantable devices such as neurostimulators demand high power resulting in small load impedance. Small coupling coefficients and load impedance limit the modulation index that can be achieved. As a consequence, the working range of the coil separation and data rate are also limited. Changing the load impedance for a longer period of time allows more time for the voltage on the receiver side to settle and hence increases the modulation index. However, this would slow down the data transmission and inevitably disturb the power transfer hence reduce the power transfer efficiency. One approach to address this trade-off is to use separate power and data links. Data rates in the region of megabit per second (Mbps) have been achieved [24]-[27] at the expense of larger device size and circuit complexity. Other approaches include using a series secondary ohmic configuration for a larger dynamic range of impedance modulation to increase the modulation index [21], or carefully choosing the timing of modulation to achieve a high data rate [23], [28]-[30].

In the presented design a passive phase-shift keying (PPSK) modulation scheme, reported in [29], has been developed. PPSK modulation uses superficially similar circuits to the conventional load impedance modulation, but imposes precise control on the timing of modulation to generate a fast transient response over the inductive link with a relatively high amplitude change. This modulation scheme is particularly suitable for biomedical implants that require a high uplink bandwidth but 


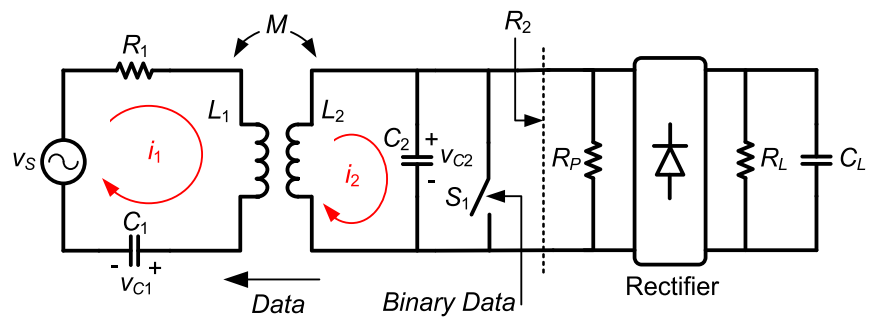

(a)

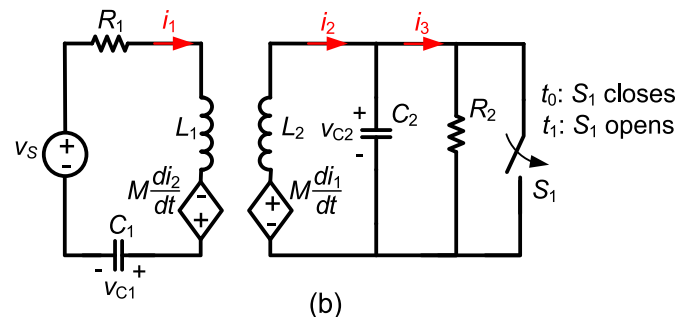

(b)

Fig. 1. Modeling PPSK modulation. (a) Simplified circuit model of an inductive link with PPSK modulation. (b) Equivalent circuit model of the inductive link.

have small coupling coefficients and low load impedances. A high modulation index can be achieved without sacrificing the data rate. By reducing the duration of the impedance change the disturbance on power transfer is also minimized. The PPSK modulator was designed for use in an integrated stimulator for a vestibular prosthesis [12] (which describes the multi-channel stimulators and the recording unit). It specifies a minimum data rate of $600 \mathrm{kbps}$ and up to $100 \mathrm{~mW}$ of power for device operation.

The focus of this paper is the telemetry design. A preliminary presentation of the PPSK modulator was reported in [28]. This paper presents in detail the theoretical principles of PPSK modulation, performance evaluation and comparison with other state-of-the-art impedance modulation techniques. Included are further circuit details of the application specific integrated circuit (ASIC), comprehensive measured results including modulation index, bit error rate, an improved data rate (up to 1.35 Mbps over a $13.56 \mathrm{MHz}$ inductive link), gain factor and efficiency of the power transfer.

The rest of the paper is organized as follows. Section II presents the theory of PPSK and evaluates its performance in simulation. Section III describes the implementation of the integrated PPSK modulator and the corresponding demodulator in the external transmitter. Section IV presents the implementation of the inductive link and the external transmitter. In Section $\mathrm{V}$ the operation of the inductive link is demonstrated with measured results. Concluding remarks are drawn in Section VI.

\section{THEORY OF PPSK}

\section{A. Principle of Modulation}

The principle of PPSK modulation [30] can be explained with reference to Fig. 1. Fig. 1(a) shows a simplified circuit model of an inductive link for PPSK modulation. In this model, $L_{1}$ and $L_{2}$ are the inductively coupled primary and secondary coils, respectively, with a mutual inductance $M$, where $M=k \sqrt{L_{1} L_{2}}$ and $k$ is the coupling coefficient between the two coils. During inductive powering, $L_{1}$ is tuned with $C_{1}$ and $L_{2}$ with $C_{2}$ at the carrier frequency. $R_{1}$ is the total serial loss resistance on the primary side, including the serial loss resistance of the primary coil and the loss resistance from the driver, $v_{S} . R_{P}$ is the parallel loss resistance of the secondary coil and $R_{L}$ is the total equivalent load resistance from the circuits driven by the secondary coil. The total load resistance seen by $L_{2}$ can be represented as $R_{2}$, where $R_{2}=R_{P} \|\left(R_{L} / 2\right)$. A switch, $S_{1}$, connects both ends of $L_{2}$ for PPSK modulation. For every binary bit "1," $S_{1}$ closes to short $L_{2}$ before it opens after a short time interval. This action creates a transient current surge in the primary loop, which can be detected by sensing the voltage peak on $C_{1}$.

An equivalent circuit model of Fig. 1(a) is shown in Fig. 1(b). Assume during operation, both $L_{1}-C_{1}$ and $L_{2}-C_{2}$ resonate at the carrier frequency $\omega_{o}$, generated by the driver $v_{S}$, a sinusoidal signal with unity peak amplitude. $v_{S}$, and the current in the primary and secondary loops, $i_{1}$ and $i_{2}$, are

$$
\left\{\begin{array}{l}
v_{S}=\sin \omega_{o} t \\
i_{1}=I_{1, \text { peak }} \sin \omega_{o} t \\
i_{2}=I_{2, \text { peak }} \sin \left(\omega_{o} t+\frac{\pi}{2}\right) .
\end{array}\right.
$$

At resonance, $i_{1}$ is in phase with $v_{S}$ and lags $i_{2}$ by $\pi / 2$, and also $i_{1}$ leads $v_{C 1}$ by $\pi / 2$ and $i_{2}$ leads $v_{C 2}$ by $\pi / 2$. Therefore, at a specific time instant $t_{0}$, where $\omega_{o} t_{0}=0$, signals $v_{S}, i_{1}$, and $v_{C 2}$ are crossing zero towards the positive phase, while $i_{2}$ is at its positive peak, $I_{2 \text {,peak }}$, with a phase of $\pi / 2$ and $v_{C 1}$ is at its negative peak with a phase of $-\pi / 2$. Similarly, at a specific time instant $t_{1}$, where $\omega_{o} t_{1}=\pi$, signals $v_{S}, i_{1}$, and $v_{C 2}$ are crossing zero again but towards the negative values, while $i_{2}$ is

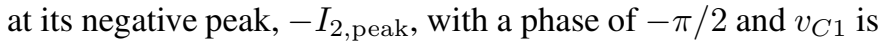
at its positive peak.

If the switch $S_{1}$ closes at $t_{0}$ to short $L_{2}$ for half a carrier

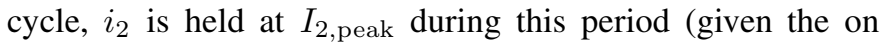
resistance of $S_{1}$ is small). $S_{1}$ is then opened after half a carrier cycle, i.e., at time instant $t_{1}$. At this instant, both $v_{S}$ and $i_{1}$ are still crossing zero towards the negative phase and $v_{C 1}$ is still at its positive peak, but $i_{2}$ is now forced to be at the positive peak instead of the negative peak in the steady-state. Denoting $i_{2, \text { steady }}\left(t_{1}\right)=-I_{2 \text {,peak }}$ as the steady-state $i_{2}$, then $i_{2}\left(t_{1}\right)=$ $i_{2, \text { steady }}\left(t_{1}\right)+2 I_{2, \text { peak }}$. It can be regarded as injecting a transient current of $i_{2 \text {,transient }}\left(t_{1}\right)=2 I_{2 \text {,peak }}$ into the secondary loop that has been running in the steady-state. The energy of the injected current contributes to a transient current surge in the primary loop. The loop response to the transient current superimposes itself onto the steady-state response, resulting in a transient voltage increase on top of the peak value of $v_{C 1}$. Using the circuit model in Fig. 1(b), the Kirchhoff equations of the transient response at $t_{1}$ are

$$
\left\{\begin{array}{l}
R_{1} i_{1}+L_{1} \frac{d i_{1}}{d t}+\frac{1}{C_{1}} \int i_{1} d t-M \frac{d i_{2}}{d t}=0 \\
L_{2} \frac{d i_{2}}{d t}+\frac{1}{C_{2}} \int\left(i_{2}-i_{3}\right) d t-M \frac{d i_{1}}{d t}=0 \\
\frac{1}{C_{2}} \int\left(i_{3}-i_{2}\right) d t+R_{2} i_{3}=0
\end{array}\right.
$$

where $i_{1}, i_{2}$, and $i_{3}$ are all transient currents as a result of injecting $i_{2 \text {,transient }}\left(t_{1}\right)=2 I_{2 \text {,peak }}$, but "transient" has been removed in the subscripts for simplicity. These transient currents are superimposed on the steady-state currents. Applying 
TABLE I

PARAMETERS OF THE INDUCTIVE LINK

\begin{tabular}{r|l}
\hline Carrier frequency & $13.56 \mathrm{MHz}$ \\
Primary coil & $0.5 \mathrm{~mm}$ diameter copper wire, \\
2 turn solenoid, 25 mm diameter \\
$L_{1}$ & $0.21 \mu \mathrm{H}$ \\
$C_{1}$ & $656 \mathrm{pF}$ \\
$R_{S}$ & $0.15 \Omega$ \\
& \\
Secondary coil & $0.315 \mathrm{~mm}$ diameter copper wire, \\
& 8 turn solenoid, 16 mm diameter \\
$L_{2}$ & $1.55 \mu \mathrm{H}$ \\
$C_{2}$ & $88.9 \mathrm{pF}$ \\
$R_{P}$ & $15.340 \mathrm{k} \Omega$ \\
\hline
\end{tabular}

Laplace transform to (2) with the initial condition of $i_{2}\left(t_{1}\right)=$ $2 I_{2, \text { peak }}$ at $t_{1}$, yields

$$
\left\{\begin{array}{l}
\left(R_{1}+s L_{1}+\frac{1}{s C_{1}}\right) I_{1}(s)-s M I_{2}(s)+2 I_{2, \text { peak }}=0 \\
s L_{2} I_{2}(s)-2 I_{2, \text { peak }}+\frac{1}{s C_{2}}\left(I_{2}(s)-I_{3}(s)\right)-s M I_{1}(s)=0 \\
\frac{1}{s C_{2}}\left(I_{3}(s)-I_{2}(s)\right)+R_{2} I_{3}(s)=0
\end{array}\right.
$$

where $s$ is the Laplace operator. From (3), rearranging $I_{1}(s)$ as a function of $I_{1, \text { peak }}$ results in a Laplace transform with a quartic denominator

$$
\begin{aligned}
& I_{1}(s) \\
& =\frac{-s \frac{2 k^{2} \omega_{o}^{2} \sqrt{1+Q_{2}^{2}}}{1-k^{2}} I_{1, \text { peak }}}{s^{4}+s^{3} \frac{\omega_{o}\left(\frac{1}{Q_{1}}+\frac{1}{Q_{2}}-\frac{k^{2}}{Q_{2}}\right)}{1-k^{2}}+s^{2} \frac{\omega_{o}^{2}\left(2+\frac{1}{Q_{1} Q_{2}}\right)}{1-k^{2}}+s \frac{\omega_{o}^{3}\left(\frac{1}{Q_{1}}+\frac{1}{Q_{2}}\right)}{1-k^{2}}+\frac{\omega_{o}^{4}}{1-k^{2}}}
\end{aligned}
$$

where $Q_{1}=\omega_{o} L_{1} / R_{1}, Q_{2}=R_{2} C_{2} \omega_{o}$, and $I_{1, \text { peak }}=I_{2, \text { peak }} /$ $\left(k \sqrt{C_{1} / C_{2}} \sqrt{1+Q_{2}^{2}}\right)$. Applying inverse Laplace transform, the $i_{1}$ natural transient can be expressed as

$$
i_{1}(t)=2\left|r^{\prime}\right| e^{-\alpha^{\prime} t} \cos \left(\omega^{\prime} t+\theta^{\prime}\right)+2\left|r^{\prime \prime}\right| e^{-\alpha^{\prime \prime} t} \cos \left(\omega^{\prime \prime} t+\theta^{\prime \prime}\right) \text {. }
$$

$i_{1}(t)$ is a pair of damped cosine waves. When $k^{2} \ll 1$, the interaction between the primary and secondary sides are minor, $\alpha^{\prime}$ and $\alpha^{\prime \prime}$ are the damping factors of the primary and secondary circuits, respectively, where $\alpha^{\prime} \approx R_{1} / 2 L_{1}$ and $\alpha^{\prime \prime} \approx 1 / 2 R_{2} C_{2} ; \omega^{\prime}$ and $\omega^{\prime \prime}$ are the damped frequencies on the primary and secondary respectively, where $\omega^{\prime} \approx \sqrt{\omega_{o}^{2}-\left(\alpha^{\prime}\right)^{2}}$ and $\omega^{\prime \prime} \approx \sqrt{\omega_{o}^{2}-\left(\alpha^{\prime \prime}\right)^{2}}$. For $k^{2} \ll 1, \omega^{\prime} \approx \omega^{\prime \prime} \approx \omega_{o}$. As $k$ increases, the interaction between the primary and secondary circuits varies the values of $\alpha^{\prime}, \alpha^{\prime \prime}, \omega^{\prime}, \omega^{\prime \prime}, r^{\prime}$ and $r^{\prime \prime}$, resulting in changes of the peak value and the rate of increase and decay of the envelope of $i_{1}(t)$.

By way of example the link parameters in Table I are specified for a vestibular prosthesis [12]. They were derived following the design procedure in Section IV.A. To evaluate $i_{1}(t)$ in (5) numerical analysis was conducted in Matlab with the parameters in Table I. Fig. 2 shows the variation of the parameters $\alpha, \omega, r$, and $\theta$ of (5) with $k$. There is a distinct change in parameter variation as $k$ approaches $k_{\text {crit }}(=0.107)$. There is always a $180^{\circ}$ phase difference between $\theta^{\prime}$ and $\theta^{\prime \prime}$. As a result, the composite waveform of $i_{1}(t)$ always has an envelope increasing to a peak and then decaying. Fig. 3 shows the resultant transient waveforms for different values of $k$. For large values of $k, \alpha^{\prime} \approx$ $\alpha^{\prime \prime}$ and $\left|r^{\prime}\right| \approx\left|r^{\prime \prime}\right|$, and an additional low frequency $\left(\omega^{\prime}-\omega^{\prime \prime}\right)$ sinewave multiplying factor develops and causes ringing.
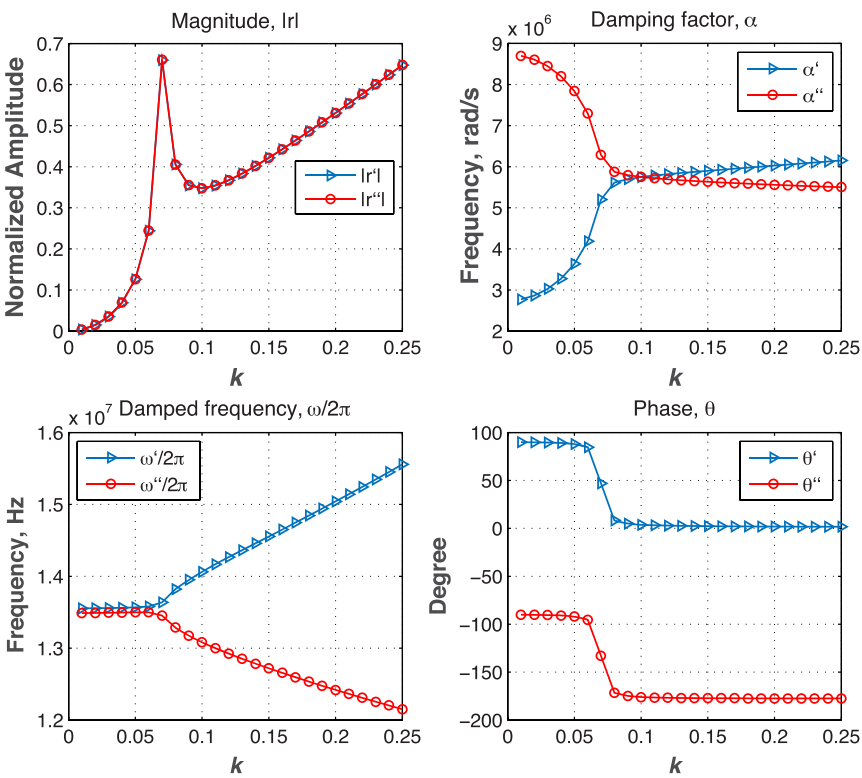

Fig. 2. Variation of the parameters of the $i_{1}$ natural transient in (5) versus coupling coefficient $k$ using the parameters in Table I.
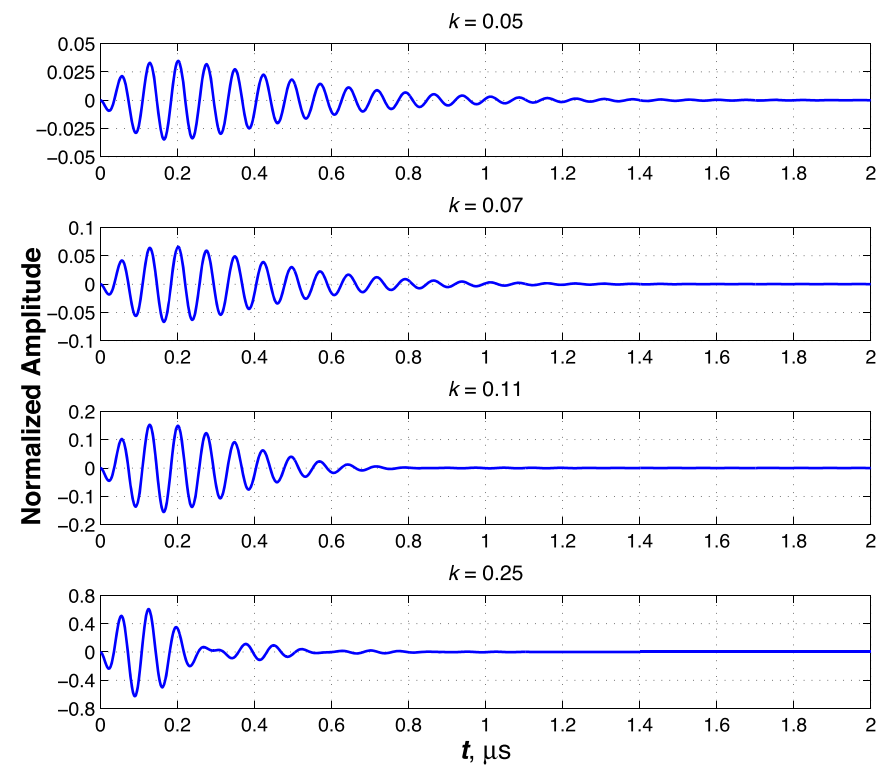

Fig. 3. Transient waveforms of the $i_{1}$ natural transient for different values of the coupling coefficient $k$ with reference to the results in Fig. 2.

\section{B. Evaluation With ADS Simulation Model}

To evaluate the performance of PPSK modulation, a simulation model based on the circuit model in Fig. 1(a) was created in Keysight Advanced Design System (ADS) v2015.01. The coupling coefficient was calculated from the coil geometry [32] using the parameters in Table I. PPSK modulation is implemented by switching on $S_{1}$, to short the secondary coil $L_{2}$. The variables in the simulations are the coupling coefficient $k$, the equivalent load resistance $R_{2}$ on the secondary coil, and the time instants at which $S_{1}$ is closed and released. For the vestibular prosthesis application, the required working range of coil separation is $5-15 \mathrm{~mm}$, corresponding to calculated coupling coefficients between 0.169 and 0.048 . 


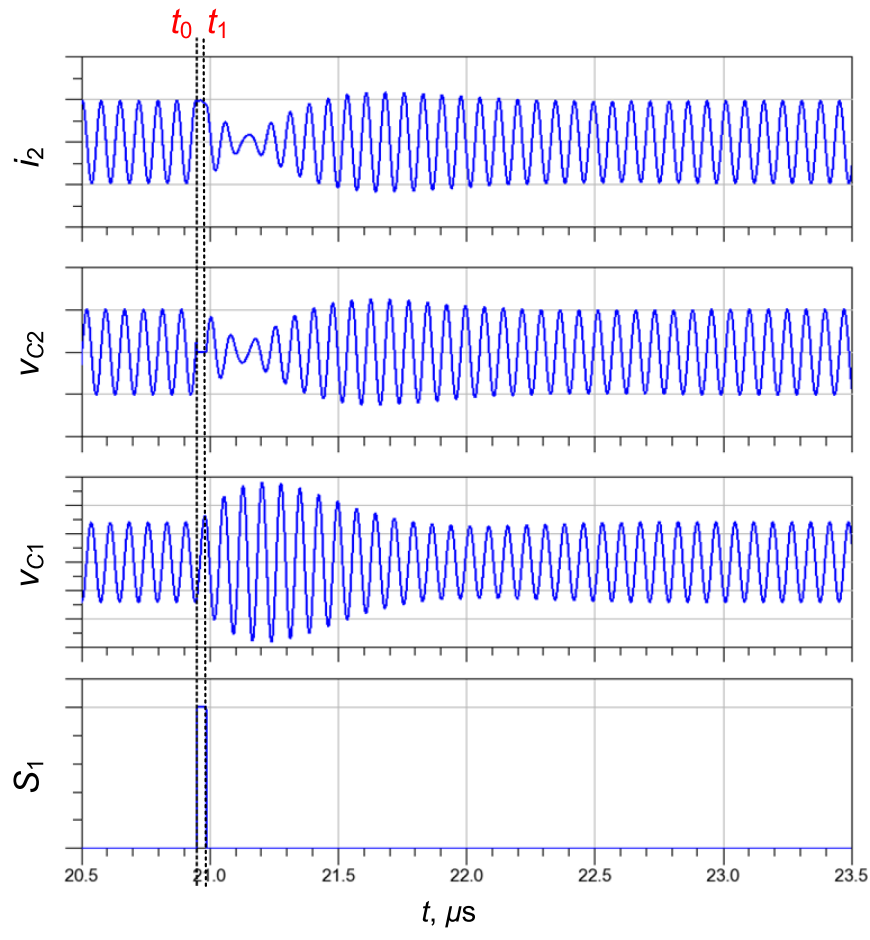

Fig. 4. Transient responses of the voltages and current in the operation of PPSK modulation. The dotted lines indicate the time instants when the secondary coil is shorted and released.

1) Transient Response: Fig. 4 shows the transient change on $i_{2}, v_{C 1}$, and $v_{C 2}$ when $S_{1}$ is closed at the instant $i_{2}$ is at its positive peak and is then released after half a carrier cycle. $k$ is set to 0.08 corresponding to $10.5 \mathrm{~mm}$ coil separation (this distance is in the middle of the expected working range of coil separation for a vestibular implant design). As shown in Fig. 4, a clear transient voltage surge can be seen on $v_{C 1}$. The rise and fall of the voltage surge shows a pattern of summation of two damped cosine waves. $v_{C 1}$ returns to its steady-state level approximately 10 carrier cycles after $S_{1}$ is closed. This gives a data rate of $1.35 \mathrm{Mbps}$ over a carrier frequency of $13.56 \mathrm{MHz}$.

2) Comparison With Other Load Modulation Schemes: The analysis in Section II.A shows that $S_{1}$ should ideally close at $t_{0}$ and open at $t_{1}$. Any timing offset from these two critical time instants will result in a lower $i_{1}$ transient and hence, lower modulation index. This is because the equivalent extra current injected into the secondary coil is at its maximum, $2 I_{2 \text {,peak }}$, when $S_{1}$ is on for half a carrier cycle, i.e., $\omega_{o} t_{1}=\pi$. When $S_{1}$ remains closed for longer, $\omega_{o} t_{2}>\pi$, where $t_{2}$ is the new time instant when $S_{1}$ opens, the new phase of the original $i_{2}$ is not opposite to the stored $i_{2}$ at $\pi / 2$, and thus the equivalent extra injected current is lowered. The performance of PPSK was compared with two other load modulation schemes: the ISO/IEC 14443Type B phase-shift keying (BPSK) load modulation scheme [33] (termed as BPSK-LSK below), and the cyclic on-off keying (COOK) scheme described in [23]. For all three schemes, the carrier frequency was set to $13.56 \mathrm{MHz}$ and the data rate to $847.5 \mathrm{kbps}$. The onset of load modulation was set to the positive zero-crossing point of $v_{C 2}$, at which point $S_{1}$ was turned on to short the secondary coil, for all the three schemes. $S_{1}$ remained on for half a carrier cycle for PPSK, one carrier cycle for COOK and eight carrier cycles for BPSK-LSK. The modulation repeated every sixteen carrier cycles. The equivalent load resistance, $R_{2}$, was set to $644 \Omega$ for all the three schemes. This value was derived from the measured $R_{L}(1344 \Omega)$ in parallel with the measured $R_{P}(15.34 \mathrm{k} \Omega)$ of the secondary coil.

Fig. 5(a) compares the modulation index of different modulation schemes. The modulation index on the primary coil is compared over the targeted working range of coil separation. The modulation index is defined as $m=(A-B) /(A+B)$, where $A$ and $B$ are the peak voltage and steady-state voltage of $v_{C 1}$, respectively. The results show that the BPSK-LSK scheme provides the highest modulation index among the three schemes, while COOK gives the lowest. PPSK produces a modulation index close to that of BPSK-LSK when $k<0.08$. Fig. 5(b) shows the influence of these three schemes on power transfer with reference to the power transfer link efficiency when no modulation is applied to the link. In contrast to the relative performance of the modulation index, COOK has the minimum disturbance on the power transfer, while BPSK-LSK reduces the link efficiency significantly. PPSK has a link efficiency slightly lower than that of COOK. Comparing the modulation index performance in Fig. 3(a), BPSK-LSK achieves the highest modulation index by having the longest duration of modulation. As a result it causes the most reduction in the power transfer. PPSK shorts the secondary coil for only half a carrier cycle. It achieves a modulation index higher than COOK by momentarily reversing the phase of LC resonance on the secondary side, and subsequently injecting extra current into the primary side. However, this phase reversal of LC resonance causes more energy loss than COOK, despite the fact that its modulation duration is shorter. Fig. 5(c) shows the minimum time required for the carrier to recover after single bit modulation with PPSK and COOK. The recovery time for both schemes increases as the coupling coefficients decreases. At strong coupling, a high data rate can be achieved as shown in [23].

The modulation strategy for specific applications can be chosen from the simulated performance. BPSK-LSK would be favourable for its highest modulation index in applications that only require occasional uplink communication with a low volume of data to transmit, such as RFID. The reduction in link efficiency from occasional disturbance on power transfer will not be significant. On the other hand, at strong coupling, COOK has the advantage of providing a high data rate with high link efficiency and acceptable modulation index. For biomedical implants with low coupling and a demand for constant uplink communication at high speed, as well as high power demand (e.g., vestibular prosthesis), PPSK is the optimal choice. At low coupling, it provides a modulation index much higher than COOK and comparable to BPSK-LSK, while it is much more power efficient than BPSK-LSK and close to COOK.

Fig. 5(d) plots the simulated modulation index against the timing error of the onset and length of the switch closure duration for PPSK modulation. The timing error is presented as the percentage of a carrier period. The modulation index is simulated with a $k$ of 0.08 and $R_{2}$ of $2 \mathrm{k} \Omega$. The highest modulation index of 0.32 occurs when both the onset and length error are zero. The results suggest a tolerance of $-15 \%$ to $10 \%$ for the length error, and $-10 \%$ to $5 \%$ for the onset error within which the modulation index remains above $90 \%$ of the peak value. 


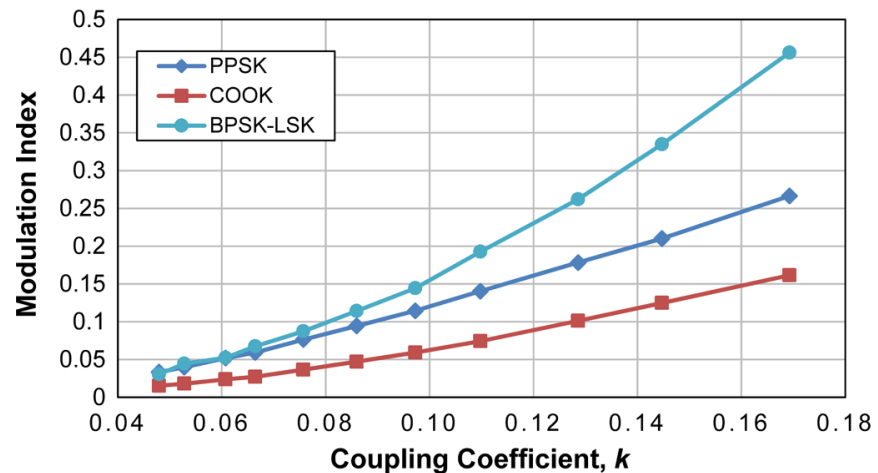

(a)

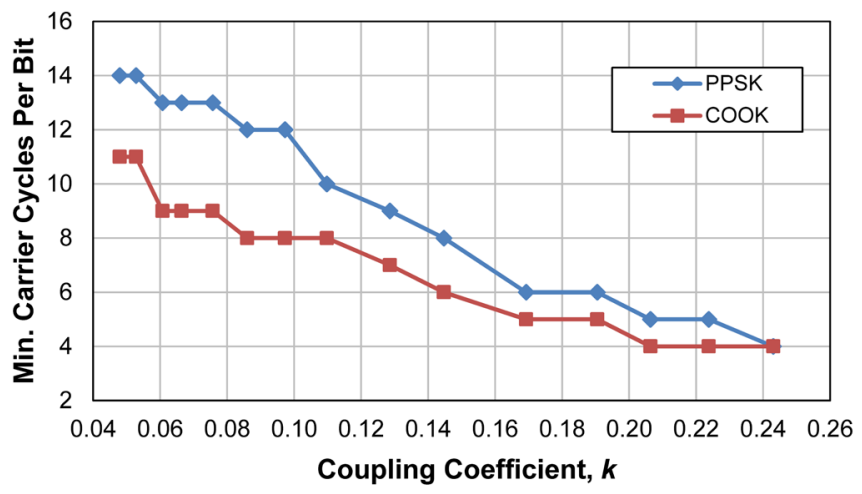

(c)

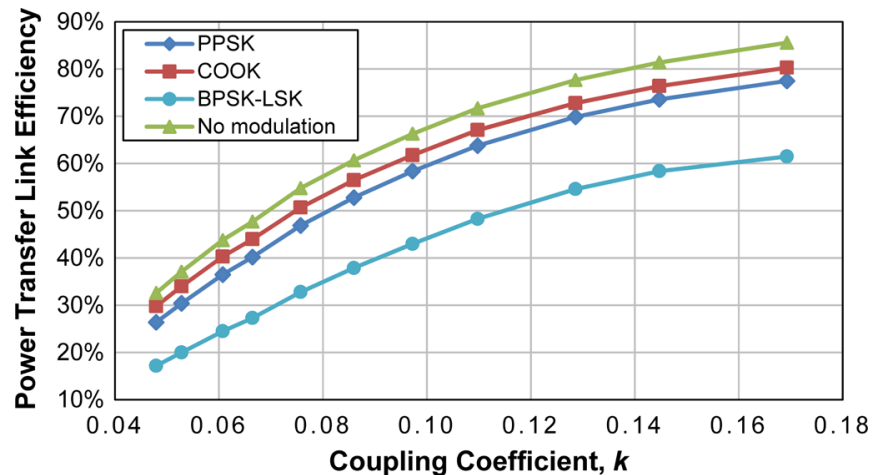

(b)

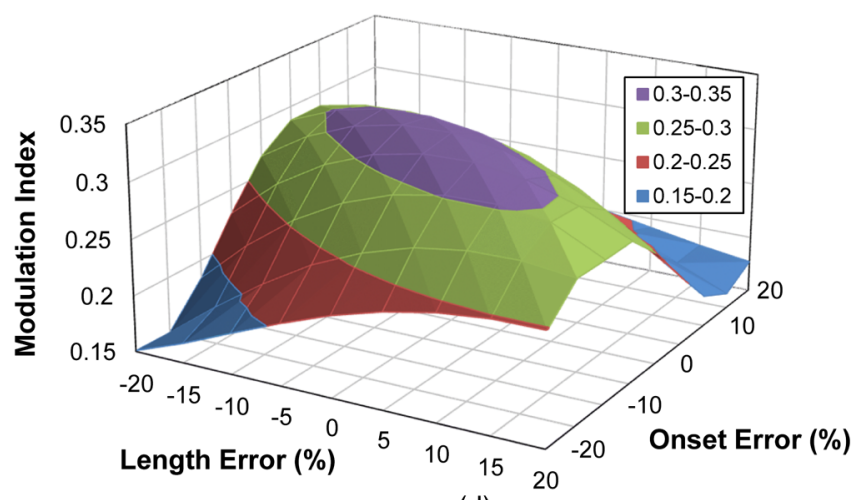

(d)

Fig. 5. Simulated performance of PPSK with comparison to COOK and BPSK-LSK, with a $R_{2}$ of $644 \Omega$, data rate of $847.5 \mathrm{kbps}$ and carrier frequency of 13.56 MHz. (a) Simulated modulation index at different coupling coefficients. (b) Simulated power transfer link efficiency at different coupling coefficients. (c) Simulated minimum number of carrier cycles per bit of PPSK and COOK for different coupling coefficients. (d) Simulated modulation index of PPSK with regard to timing offset of both the start and duration of the switch closure.

\section{Implant}

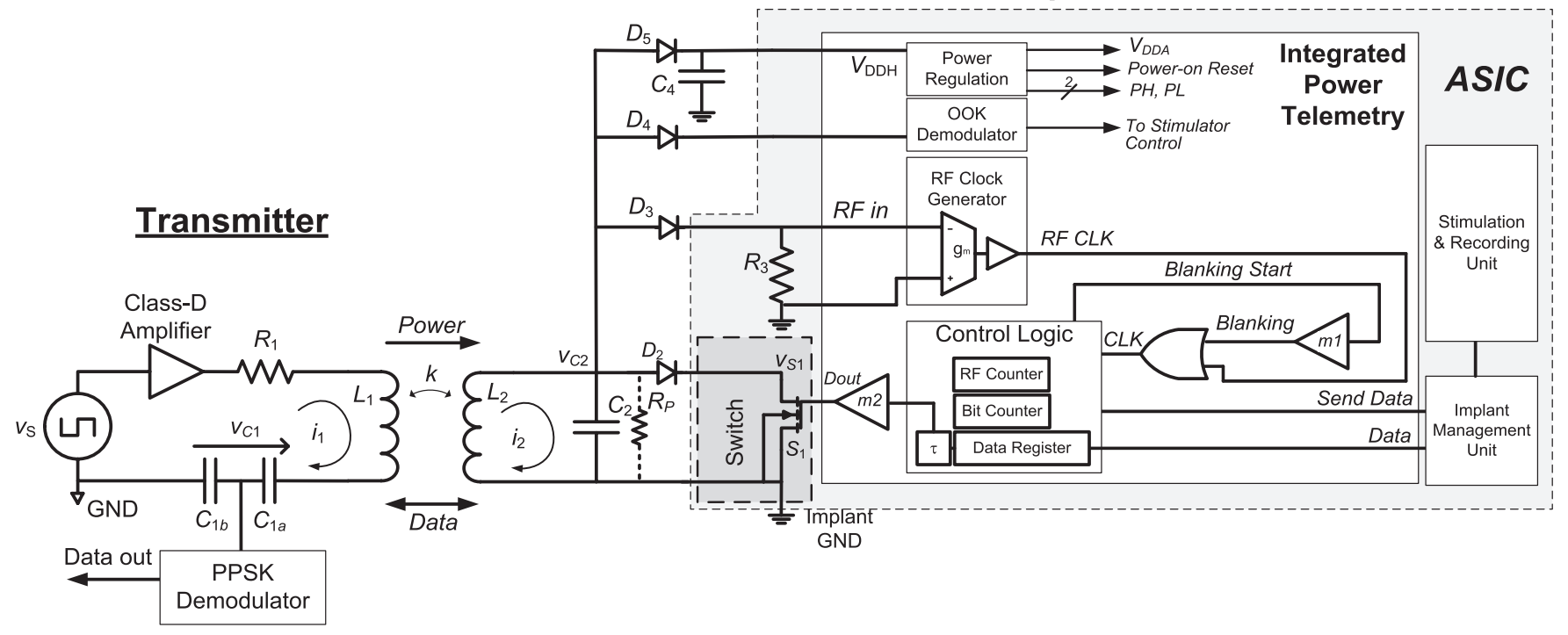

Fig. 6. System architecture of the power telemetry with PPSK modulation. The integrated components are in the area labeled ASIC.

\section{IMPLEMENTATION OF THE TELEMETRY}

\section{A. System Overview}

A power telemetry with an integrated PPSK modulator was implemented as part of an implantable stimulator for vestibular prosthesis (requiring up to $100 \mathrm{~mW}$ power delivery and 600 kbps uplink data communication [12]). The system archi- tecture is shown in Fig. 6 with emphasis on the power and data telemetry. Power transfer is via the inductive link with its tuning network, including $L_{1}, C_{1 a}, C_{1 b}$ on the primary side and $L_{2}$, $C_{2}$ on the secondary side. The tuning network is adjusted to the carrier frequency of $13.56 \mathrm{MHz}$. Downlink data transmission uses on-off keying (OOK) while uplink employs PPSK. The implant subsystem was integrated in an ASIC as highlighted 


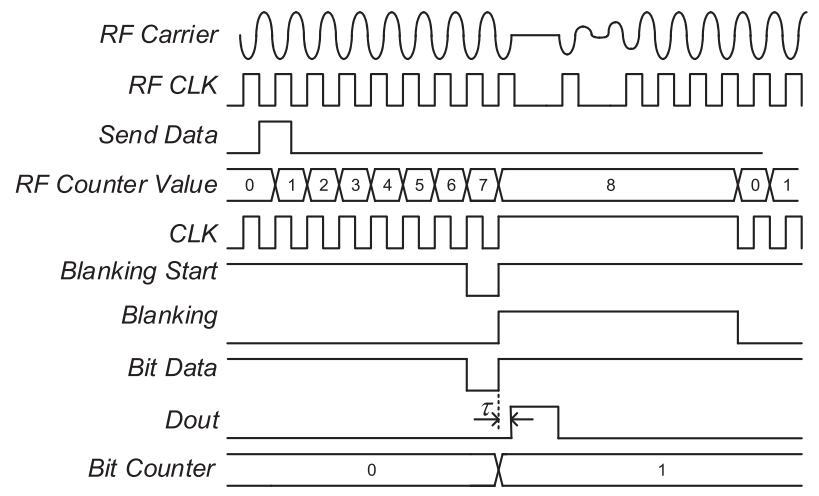

Fig. 7. Timing sequence of the control logic.

in Fig. 6. Diodes $D_{2}-D_{5}$ are glass case Schottky diodes and $C_{4}$ is a $10 \mu \mathrm{F}$ tantalum capacitor. The ASIC includes a power regulator providing a stable high voltage, $V_{\mathrm{DDH}}$, of $12 \mathrm{~V}$ for the stimulator output stage and a stable low voltage, $V_{\mathrm{DDA}}$, of $5 \mathrm{~V}$ for the rest of system, comprising a PPSK modulator, an OOK demodulator, an implant management unit and a stimulation and recording unit. Details of the management unit and stimulation and recording circuits were presented in [11], [12]. The carrier is generated by a class-D power amplifier in the external transmitter. The voltage developed in the resonant tank $L_{2}-C_{2}$ is rectified by a half-wave rectifier, $D_{5}-C_{4}$, for the power regulator. The signal on the secondary coil is connected to the OOK demodulator and the PPSK modulator.

\section{B. PPSK Operation}

The PPSK modulation is managed by a control logic that operates on a clock extracted from the carrier by the RF clock generator. The control logic contains a shift register for the data, two counters and a delay block. The shift register streams out data received from the implant management unit at a speed defined by the RF counter. The timing sequence of the modulation control is shown in Fig. 7. A Send Data signal from the implant management unit triggers the modulation procedure in which the RF counter starts counting the $R F C L K$ pulses generated from the $R F$ Carrier. At the overflow value of the counter ( 7 in Fig. 7 for a bit rate of $847.5 \mathrm{kbps}$ ), if the current bit is logic " 1 ," a Bit Data pulse is generated to trigger the monostable $m 2$ to generate a pulse on Dout. This pulse turns on switch $S_{1}$ for PPSK modulation. $S_{1}$ was implemented by a high voltage NMOS transistor with dimensions $W / L=1995 \mu \mathrm{m} / 1.3 \mu \mathrm{m}$, yielding an on resistance of $17 \Omega$.

Since the received carrier disappears for a few cycles when modulation occurs due to shorting the secondary coil, RF CLK becomes momentarily irregular during modulation, as shown in Fig. 7. To maintain regularity and constant timing between bits, a blanking mechanism was implemented. After the $R F$ Counter Value reaches its overflow value, a Blanking Start pulse is generated to trigger the monostable $m 1$, which generates a blanking pulse of about $570-590 \mathrm{~ns}$, sufficiently longer than the required time for the carrier to recover. This pulse blanks the clock to the control logic from $R F C L K$, keeping $C L K$ static until $R F C L K$ returns to regular action. The data rate is programmable by setting the overflow value of the RF counter. This value is set by the external transmitter during system initialisation. Once the counter exceeds this value, it resets and starts the next modulation period, where the shift register streams out the next bit and the bit counter increments by 1 . The overall period for each bit is the sum of the length of the blanking pulse and the number of carrier cycles set by the overflow value.

As discussed in Section II, the timing of switching on and off $S_{1}$ is critical for achieving the maximum modulation index. A delay cell, $\tau$, was inserted at the input of $S_{1}$ to align the switch onset. The length of the delay is a sum of the delay from the monostable $m 2$, the control logic and the RF clock generator. Its value was derived from Monte-Carlo simulation to ensure the onset error is within $10 \%$ of one period of the carrier. In addition, $m 2$ is made programmable so that the length of the $S_{1}$ pulse can be fine-tuned to reduce the length error.

\section{Monostable}

The circuit implementation and operation of the two monostable units ( $m 1$ and $m 2$, Fig. 6) are shown in Fig. 8. Fig. 8(a) shows the overall architecture of the monostable. In operation, a rising edge at the input $I N$ switches the output OUT to "1" and turns on the current source $I_{C}$ to charge the capacitor $C$. When the voltage on $C$ reaches a high threshold value, $V_{\mathrm{hi}}$, the D-flip flop is reset. As a result, its output $Q$ becomes " 0 " and $I_{C}$ is turned off and the current sink, $I_{D}$ is switched on to discharge $C$. Once the voltage on $C$ drops to $V_{\text {low }}, O U T$ is switched back to " 0 ." The circuit stays in this state until the next pulse on $I N$ arrives. The width of the output pulse on $O U T$ is decided by the charging and discharging current value, the capacitance of $C$ and the threshold values of $V_{\mathrm{hi}}$ and $V_{\text {low }} . V_{\text {hi }}$ and $V_{\text {low }}$ are the threshold voltages of inverter " $h i$ " and "low" in Fig. 8(a), respectively. The charging time, $t_{c}$, and the discharging time, $t_{d}$, are

$$
\begin{aligned}
t_{c} & =\frac{C V_{\mathrm{hi}}}{I_{C}} \\
t_{d} & =\frac{C\left(V_{\mathrm{hi}}-V_{\mathrm{low}}\right)}{I_{D}} .
\end{aligned}
$$

The threshold voltage of a simple CMOS inverter is [34]

$$
V_{\mathrm{th}}=\frac{V_{\mathrm{th} N} \sqrt{\frac{\beta_{N}}{\beta_{P}}}+V_{\mathrm{DDA}}-V_{\mathrm{th} P}}{1+\sqrt{\frac{\beta_{N}}{\beta_{P}}}}
$$

where $\beta_{N} / \beta_{P}$ is the transconductance ratio of the NMOS and PMOS transistors; $V_{\mathrm{th} N}$ and $V_{\mathrm{th} P}$ are the threshold voltages.

The current source and sink of $m 2$ are programmable as shown in Fig. 8(c). $I_{C}$ is mirrored with transistors M15, M25, M27, M28, M36, and M37 to generate a fixed current of $136 \mu \mathrm{A}$. $I_{D}$ consists of two parts: a fixed current $I_{\text {base }}$ of $80 \mu \mathrm{A}$ mirrored with M15, M19, M22, and M24, and a tuning current $I_{\text {control. }} . I_{\text {control }}$ is eight times the programmable current $I_{\text {program }}$ generated from $M_{1}-M_{14}$. The variation range of $I_{\text {control }}$ is between $0 \mu \mathrm{A}$ and $60 \mu \mathrm{A}$ with a step size of $4 \mu \mathrm{A}$. $I_{\text {control }}$ is programmed by the digital values of $A 0, A 1, A 2$, and $A 3$ that are set by the external transmitter. Charging and discharging are controlled by signal $Q n$ that turns on or off of the transistor switches M21, M23, M33, and M38. 


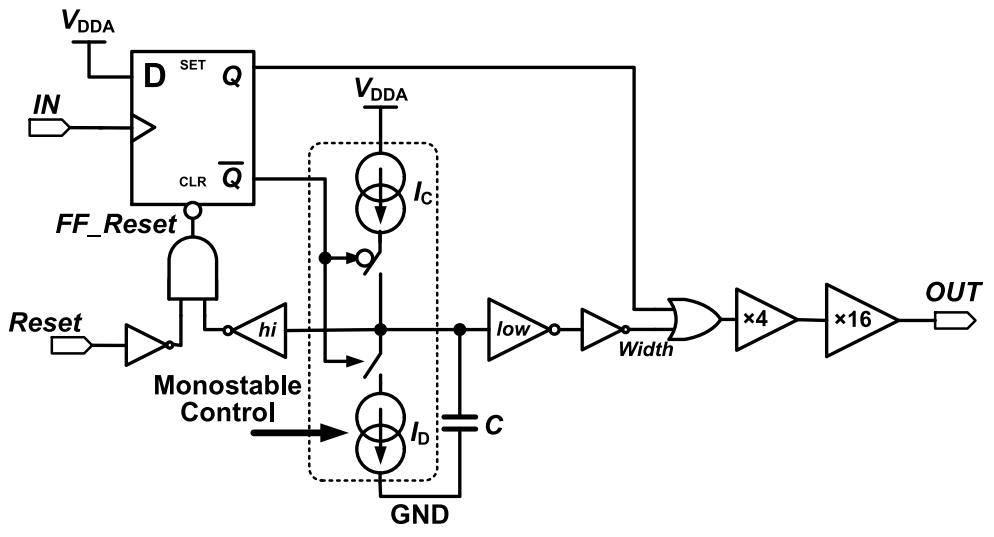

(a)

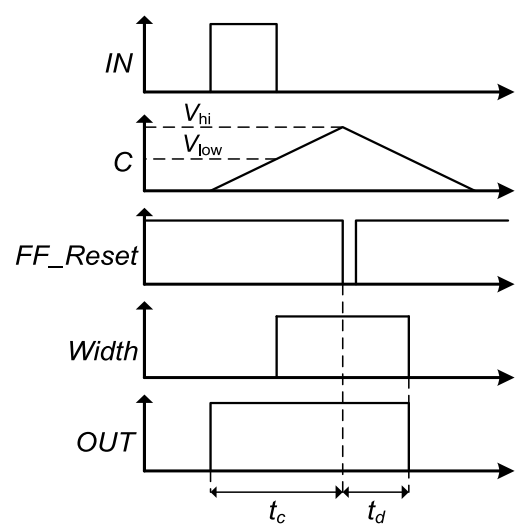

(b)

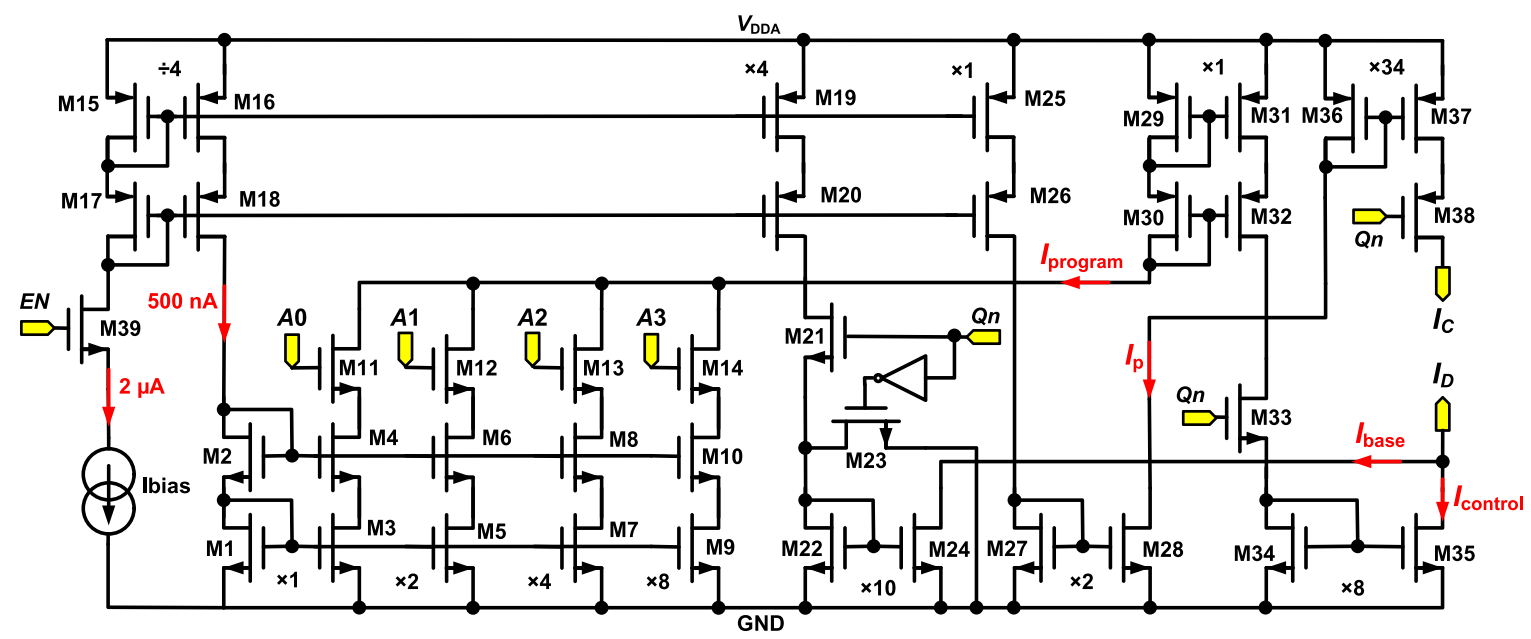

(c)

Fig. 8. Programmable monostable implementation. (a) Monostable schematic. (b) Operation of the monostable. (c) Programmable current source.

The value of the capacitor $C$ in $m 2$ is $1.68 \mathrm{pF}$ (included in the ASIC). The transistor dimensions of the inverters are: for "high," PMOS $W / L=4 \mu \mathrm{m} / 10 \mu \mathrm{m}$ and NMOS $W / L=1 \mu \mathrm{m} / 10 \mu \mathrm{m}$; for "low," PMOS $W / L=3 \mu \mathrm{m} / 10 \mu \mathrm{m}$ and NMOS $W / L=$ $5 \mu \mathrm{m} / 10 \mu \mathrm{m}$. The duration of the $m 2$ output pulse $(O U T)$ is between $107 \mathrm{~ns}$ and $116.3 \mathrm{~ns}$ with a step size of $0.62 \mathrm{~ns}$. This is suitable for matching 1.5 cycles of the $13.56 \mathrm{MHz}$ carrier. The choice of 1.5 cycles instead of 0.5 cycle is to allow a larger $C$ so that the effect of the process variation is reduced. A switch closure of 1.5 carrier cycles generates the same PPSK modulation performance because the switch release time is still at the opposite phase of the switch closure onset, while the current loss in the secondary loop during switch closure is low given the high parallel loss resistance of the coil and the low on resistance of the switch.

The implementation of the monostable $m 1$ is the same architecture as that in Fig. 8(a) but with $I_{C}=40 \mu \mathrm{A}, I_{D}=48 \mu \mathrm{A}$ and $C=5.73 \mathrm{pF}$ (included in the ASIC).

\section{RF Clock Generator}

To extract the carrier frequency from the RF signal in the digital domain a level shifting circuit was implemented as shown in Fig. 9. The differential amplifier generates a pulse when the carrier amplitude is above $0 \mathrm{~V}$. This results in a square wave at the output of the differential amplifier. The following

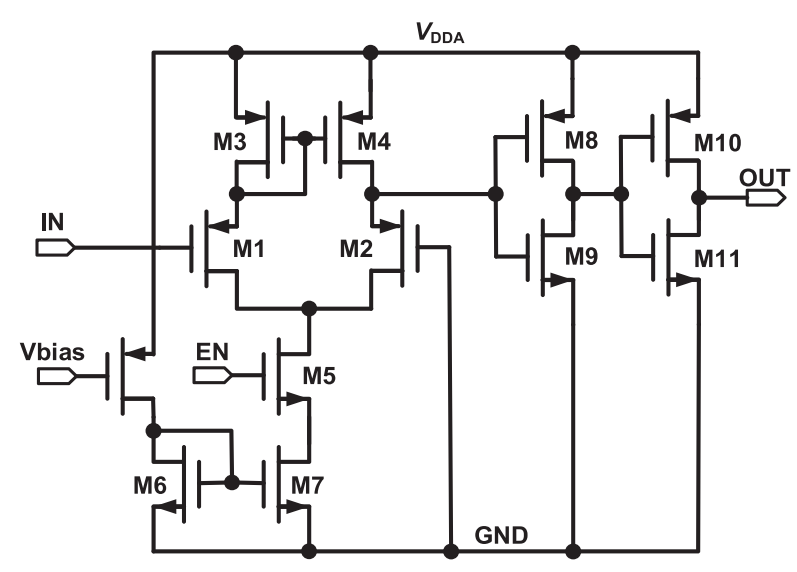

Fig. 9. RF clock generator.

inverters generate a square wave compatible digital signal to serve as the clock for the PPSK control logic.

\section{E. OOK Demodulator}

The implant receives control settings from the external transmitter via the inductive link using OOK modulation with a data rate of $400 \mathrm{kbps}$. The circuit of the OOK envelope detector to extract the signal from the modulated carrier is shown in Fig. 10. As the recovery time of the carrier varies with the load and coupling coefficient, the time constant of the $R C_{d}$ peak 


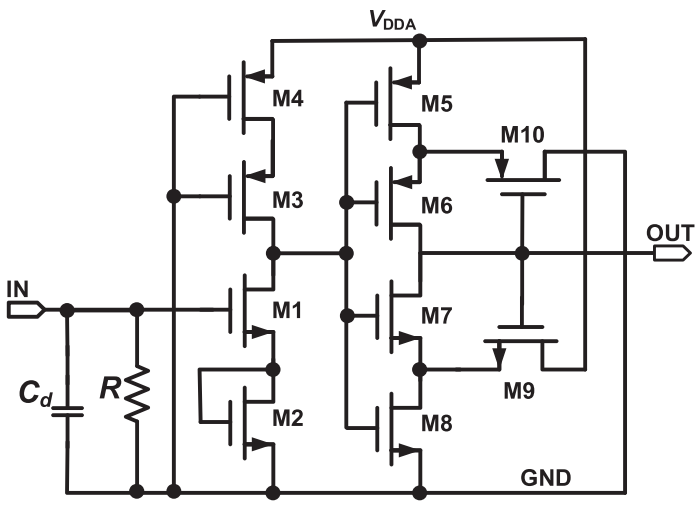

Fig. 10. Implementation of the OOK demodulator.

detector should be set to a larger value than the worst case rise time of the carrier. The implemented $R$ is $44.9 \mathrm{k} \Omega$ and $C_{d}$ is $2.8 \mathrm{pF}$. Transistors M5-M10 form a Schmitt trigger to generate a digital output from the peak detector.

\section{F. Power Regulator}

The power regulator module includes a voltage monitoring unit, a voltage regulator and a power-on reset. The voltage monitoring unit reports to the external transmitter when the received voltage is too high or too low (by respectively setting $P H$ and $P L$; see Fig. 6) and the driving voltage $V_{\text {driver }}$ on the class-D amplifier (see Fig. 11) is adjusted accordingly. The voltage regulator generates a stable $5 \mathrm{~V}$ supply for the implant electronics. The voltage regulator and the power-on reset are implemented in standard circuit architecture as described in [34].

\section{EXTERNAL TRANSMITTER AND COMMUNICATION PROTOCOL}

\section{A. Link Design}

The diameter of the secondary coil and the coil separation are mainly defined by the surgical constraints. In the example of the vestibular application, the layout of the implant is similar to commercial cochlear implants [35], where the secondary coil locates alongside a hermetically sealed hybrid. Both the coil and hybrid are encapsulated with medical grade silicone rubber. The diameter of the secondary coil $L_{2}$ was set to $16 \mathrm{~mm}$. The working range of the coil separation takes into account the thickness of the skin behind the ear where the device will be implanted, and the thickness of the secondary coil after encapsulation. It is set between $5 \mathrm{~mm}$ and $15 \mathrm{~mm}$, while the gain factor of the power transfer peaks around 7-10 $\mathrm{mm}$. To accommodate misalignment due to relative movement between the coils on implantation, the diameter of the primary coil $L_{1}$ was set to $25 \mathrm{~mm}$ to ensure $100 \%$ overlap.

The gain factor of the power transfer (refer to Fig. 1) can be expressed as [36]

$$
A_{G}=\frac{\left|v_{C 2}\right|}{\left|v_{S}\right|}=\frac{\sqrt{\frac{R_{2}}{R_{1}}}}{\left(\frac{k_{c}}{k}+\frac{k}{k_{c}}\right)}
$$

where $k_{c}$ is the critical coupling coefficient

$$
k_{c}=\sqrt{\frac{C_{1} R_{1}}{C_{2} R_{2}}} .
$$

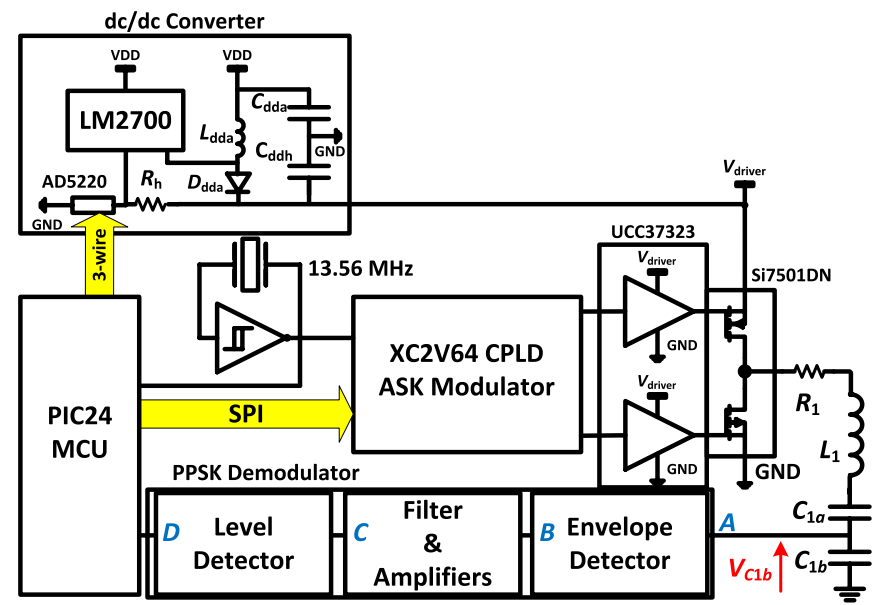

Fig. 11. Architecture of the external transmitter.

Equation (9) indicates that $A_{G}$ peaks when $k_{c}=k$. Therefore, the values for $C_{1}, C_{2}, R_{1}$, and $R_{2}$ are bounded by (9) so that $k_{c}=k$ at $8 \mathrm{~mm}$ coil separation. Among the variables, $R_{1}$ is a combination of the primary coil serial loss resistance and the loss resistance of the power amplifier and $R_{2}=R_{P} \|\left(R_{L} / 2\right)$. For this design, $R_{1}$ is $1.15 \Omega$, while the load resistance of the implant, $R_{L}$, is about $1344 \Omega$ from measurements. The carrier frequency is $13.56 \mathrm{MHz}$. Based on the values of $R_{1}, R_{2}$, the geometry of the coils and the carrier frequency, $L_{1}$ and $L_{2}$ were chosen to satisfy $k_{c}=k$ at $8 \mathrm{~mm}$ coil separation, where the value of $k$ was derived using the procedure in [30]. The link parameters are listed in Table I. According to these parameters, $k$ is between 0.048 and 0.169 , and $k_{c}$ is 0.107 at $8 \mathrm{~mm}$ coil separation.

\section{B. External Transmitter}

The architecture of the external transmitter is shown in Fig. 11. A class-D power amplifier was constructed with the primary LC tank driven by a pair of power switches built with two complementary power MOSFETs, Si7501DN, and a dual MOSFET driver UCC37323. A XC2V64 CPLD (complex programmable logic device) operates the power switches at a switching frequency of $13.56 \mathrm{MHz}$. A microcontroller (MCU) controls the power transfer level by adjusting the supply voltage of the class-D amplifier. According to the feedback voltage measurement from the implant, the MCU programs a digital potentiometer, AD5220, in the dc/dc converter that supplies the class-D amplifier. The MCU also generates control settings for the implant. These settings are sent via SPI (serial peripheral interface) to the CPLD, where they are packed into frames with cyclic redundancy check (CRC) code generated and attached. The CPLD also shifts the frames into a bit-stream and performs OOK modulation on each bit. The modulation is implemented by switching on or off the driving signal to the power switches at 400 kbps. Logic " 1 " switches off the carrier while logic "0" keeps the carrier unchanged.

The uplink data stream is demodulated in the PPSK demodulator. A simple capacitive voltage divider, $C_{1 a}$ and $C_{1 b}$, scales down the modulated carrier in the primary LC tank to the dynamic input range of the amplifiers in the filtering and 


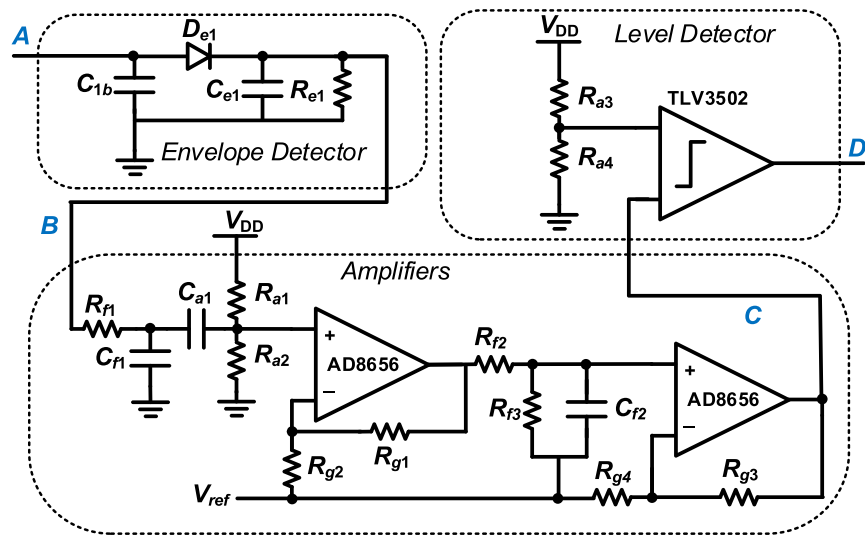

Fig. 12. Schematic of the PPSK demodulator.

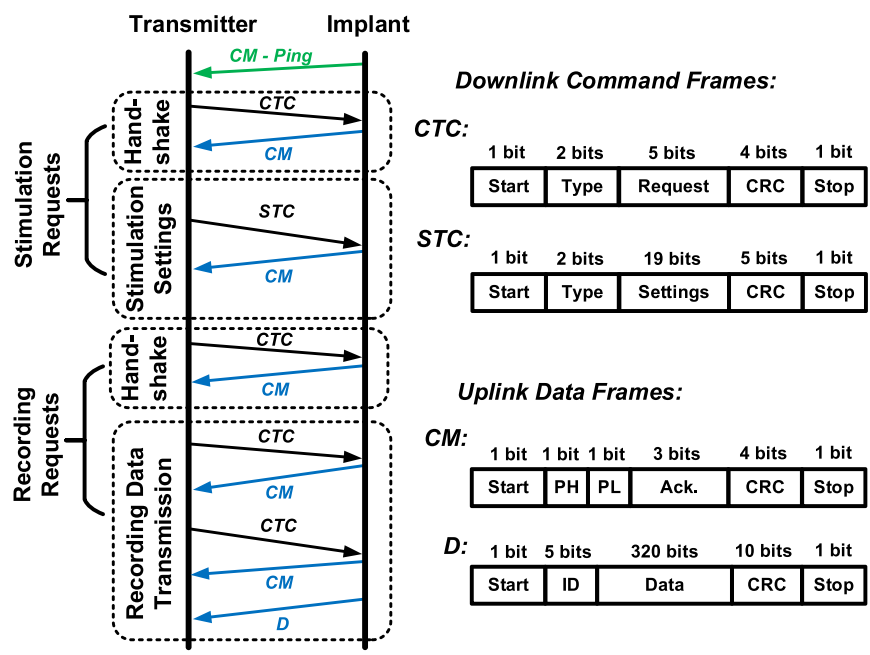

Fig. 13. Communication protocol and the frame structures.

amplifying stage. The ratio between capacitors $C_{1 a}$ and $C_{1 b}$ is $1: 8.57$. The voltage on $C_{1 b}$ is applied to the PPSK demodulator, which comprises an envelope detector, a filter and amplifier stage and a level detector. The circuit details of the three stages are shown in Fig. 12. The envelope detector extracts the voltage transient on $C_{1 b}$ from the input. The time constant of the $R_{e 1}-C_{e 1}$ network was set to be higher than the carrier period (73.7 ns) but lower than a single bit width $(>740 \mathrm{~ns})$. A RC low pass filter $\left(R_{f 1}-C_{f 1}\right)$ with a cut-off frequency of $2 \mathrm{MHz}$ attenuates the carrier frequency components from the output of the envelope detector. The ac components in the filtered signal are applied to a two-stage amplifier with a dc bias at the midsupply range. The two-stage amplifier consists of two AD8656 op-amps and a low-pass filter between the two amplifiers. The amplified signal is translated into a digital bit-stream by a level detector implemented with a comparator TLV3502.

\section{Communication Protocol}

The external transmitter and the implant communicate over the inductive link following a half-duplex protocol shown in Fig. 13. There are two types of downlink frame, CTC and STC, and two types of uplink frame, $C M$ and $D$. All frames begin with a logic "1" as Start and end with a logic "0" as Stop, so that the RF carrier remains intact when there is no communication.

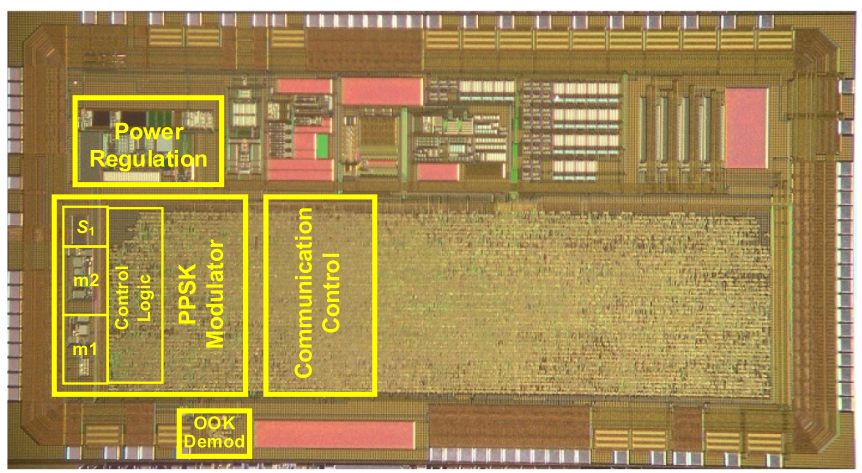

Fig. 14. ASIC microphotograph with the building blocks of the telemetry highlighted.

When the implant is powered up, it sends $C M$ frames to the transmitter repetitively every $250 \mu$ s as Ping signals until the transmitter responds. The procedure for stimulation, recording and housekeeping requests consists of two stages. The transmitter firstly sends a CTC frame for handshake. The Request section in the frame defines the request. The implant completes the handshake with a $C M$ frame as acknowledgement. The transmitter then sends a STC frame with the settings for the request, such as the pulse profile and channel for stimulation or recording window length and channel selection. Details of the stimulation and recording settings are described in [12]. The housekeeping request includes settings for the PPSK data rate control and programmable monostable. The settings can be sent over multiple $S T C$ frames. The implant acknowledges each $S T C$ frame with a $C M$ frame. The Ack. section in the $C M$ frame informs the transmitter whether the $S T C$ frame was correctly received or not. For a recording request, the transmitter repeatedly sends $C T C$ frames to check if the 320-bit buffer in the implant, corresponding to a $1.6 \mathrm{~ms}$ neural recording window length, has been filled up with recorded data. The implant reports the buffer status in the Ack. section of the $C M$ frames. Once the buffer is full, the implant starts transmitting data in a $D$ frame. All the frames contain CRC for error detection at the receiving end. The length of CRC depends on the length of a frame. Each $C M$ frame also contains two power bits, $P H$ and $P L$, for reporting whether or not the received voltage is too high or too low, so that the transmitter can adjust the power transfer accordingly.

\section{Measured Results}

The ASIC was implemented in 0.6- $\mu \mathrm{m}$ high voltage CMOS technology. Fig. 14 shows the die microphotograph with the building blocks of the telemetry highlighted. Table II lists features and measured performance. For the measurements, the external transmitter and the implant electronics were mounted on two separate printed circuit boards (PCBs). The implant electronics includes the ASIC shown in Fig. 14, the auxiliary discrete components for the telemetry $\left(D_{2}-D_{5}, C_{2}\right.$, and $C_{4}$ shown in Fig. 6), and resistors used as equivalent electrode load impedances [12]. The primary and secondary coils as specified in Table I were connected closely to the two PCBs. The two coils were mounted onto two parallel panels in a coaxial position. The distance between the two coils was adjusted by moving one panel along the axis. 
TABLE II

SumMARY OF FEATURES AND PERORMANCE

\begin{tabular}{|c|c|}
\hline Technology & X-FAB 0.6- $\mu \mathrm{m}$ HV CMOS \\
\hline Die size & $\begin{array}{l}\text { ASIC: } 21.42 \mathrm{~mm}^{2} \text { (including the telemetry, } \\
\text { implant management, stimulation and } \\
\text { recording circuits) }\end{array}$ \\
\hline Supply voltage & $\begin{array}{l}5 \mathrm{~V} \text { (digital circuits, PPSK modulator, } \\
\text { OOK demodulator, recording unit) } \\
12 \mathrm{~V} \text { (stimulator output stage) }\end{array}$ \\
\hline \multicolumn{2}{|l|}{ Telemetry } \\
\hline Carrier frequency & $13.56 \mathrm{MHz}$ \\
\hline Working distance & $5-15 \mathrm{~mm}$ \\
\hline Uplink modulation & PPSK \\
\hline Uplink data rate & Up to $1.35 \mathrm{Mbps}$ \\
\hline Uplink BER & $\begin{array}{l}<1.01 \times 10^{-6} \text { at } 847.5 \mathrm{kbps} \\
<1 \times 10^{-5} \text { at } 1.35 \mathrm{Mbps}\end{array}$ \\
\hline Downlink modulation & OOK \\
\hline Downlink data rate & $400 \mathrm{kbps}$ \\
\hline Power transfer & Up to $100 \mathrm{~mW}$ \\
\hline $\begin{array}{l}\text { Power transfer link } \\
\text { efficiency }\end{array}$ & $66.4 \%$ without PPSK, $60.64 \%$ with PPSK ${ }^{* *}$ \\
\hline
\end{tabular}

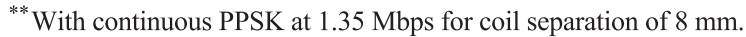

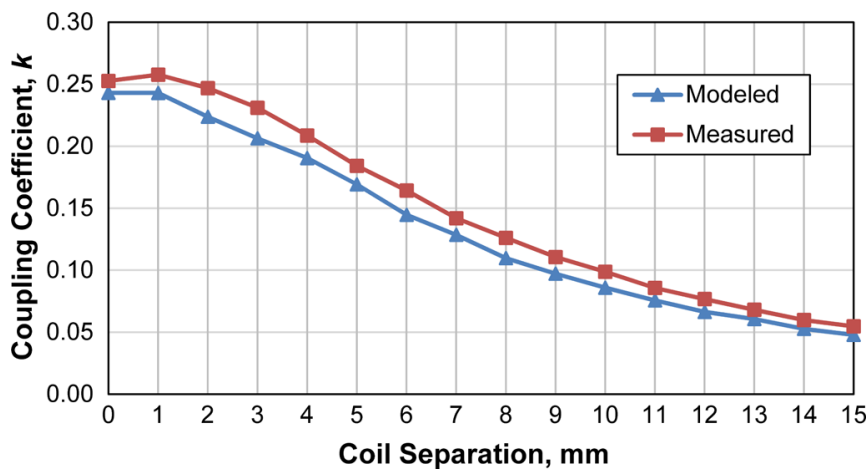

Fig. 15. Modeled and measured coupling coefficient for different coil separations.

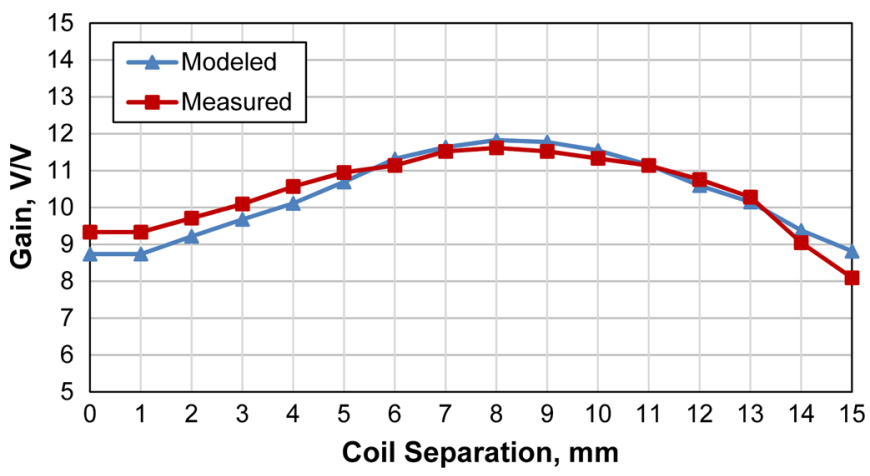

Fig. 16. Modeled and measured gain factor for different coil separations.

\section{A. Power Transfer}

The coupling coefficients at different coil separations were calculated from measured mutual inductance and compared to those modeled shown in Fig. 15. The modeled coupling coefficients in the figure were derived from the coil geometry using the procedure in [32]. Fig. 16 shows the gain factor against coil separation of the inductive link driving the vestibular prosthesis with $R_{L}=1344 \Omega$. The measured gain factor matches the result derived from (9) using the modeled coupling coefficients. The gain factor peaks around $8 \mathrm{~mm}$ coil separation, agreeing with the calculated critical coupling position.

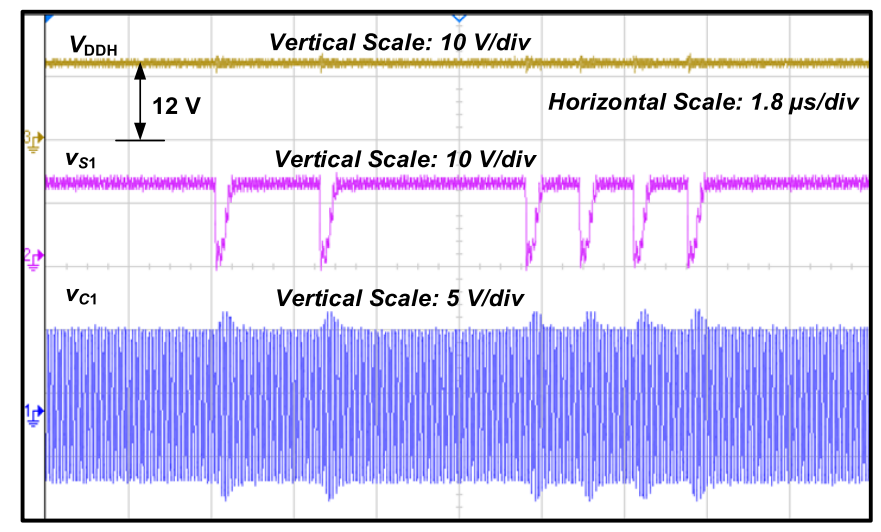

(a)

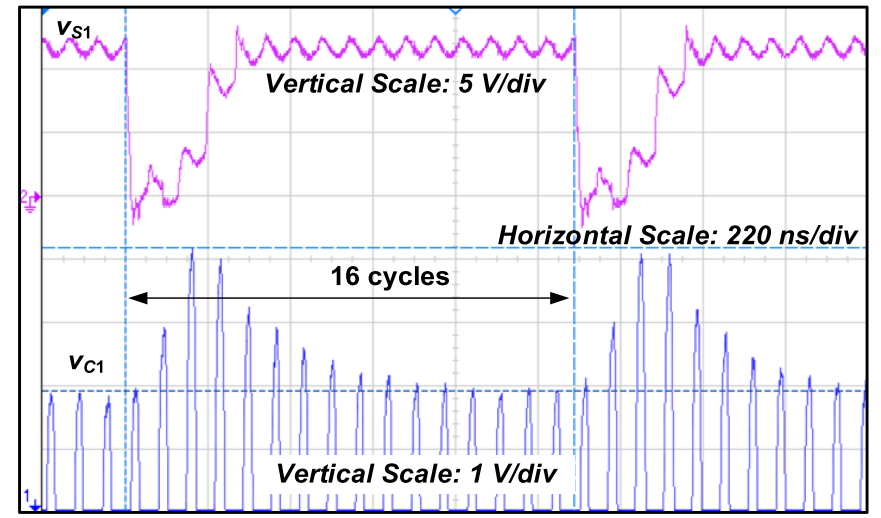

(b)

Fig. 17. (a) Oscilloscope screenshot of the transmission of a CM packet with PPSK modulation at a coil separation of $7 \mathrm{~mm}$. (b) Zoomed in view of two consecutive bits of " 1. "

\section{B. PPSK Modulation and Demodulation}

Fig. 17 shows the performance of the PPSK modulator with the vestibular implant sending a $C M$ frame over the inductive link. The coil separation was $7 \mathrm{~mm}$, corresponding to a measured $k$ of around 0.14 . A $C M$ frame with an 11-bit sequence (10100011110) was transferred over the inductive link. In Fig. 17(a), the top trace is the power regulator output $V_{\mathrm{DDH}}$ that supplies the implant. $V_{\mathrm{DDH}}$ is a stable de voltage of $12 \mathrm{~V}$. The middle trace is the voltage $v_{S 1}$ recorded at the drain of the switch $S_{1}$, as shown in Fig. 6. $S_{1}$ is shorted at every logic " 1 " bit for 1.5 carrier cycles. As a result, a transient voltage surge on $v_{C 1}$ can clearly be seen in the bottom trace in Fig. 17(a). Fig. 17(b) shows a zoom-in view of $v_{S 1}$ and $v_{C 1}$ when two consecutive logic " 1 " bits are transmitted. Switching of $S_{1}$ causes a transient voltage surge on $v_{C 1}$ that lasts for nine carrier cycles before it returns to the steady-state. In order to allow sufficient settling time over the entire working range of 5-15 $\mathrm{mm}$ for the coil separation, each bit of the uplink data lasts for sixteen carrier cycles before transmitting the next bit. This corresponds to data rates up to $847.5 \mathrm{kbps}$.

The measurement was repeated with different coil separations, and the recorded modulation index is shown in Fig. 18 alongside the simulated modulation index from ADS with $R_{2}=644 \Omega$. As shown, the measured values approximately agree with the simulation, decreasing with the increase of the coil separation. 


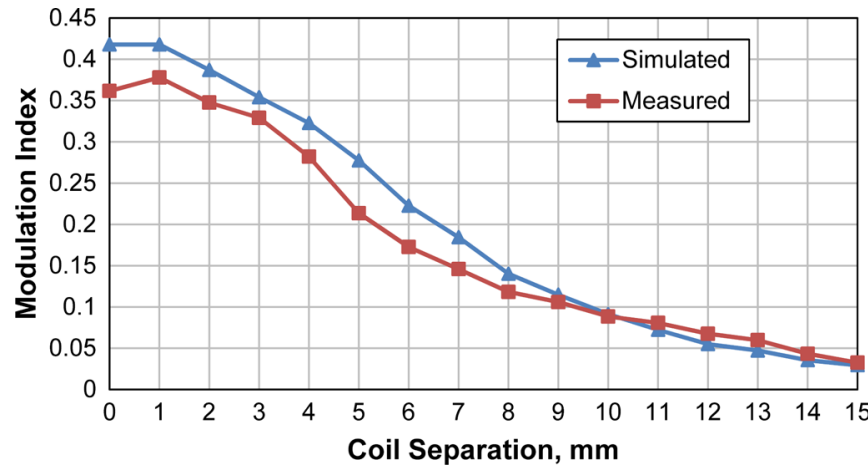

Fig. 18. Simulated and measured variation in modulation index for different coil separations.

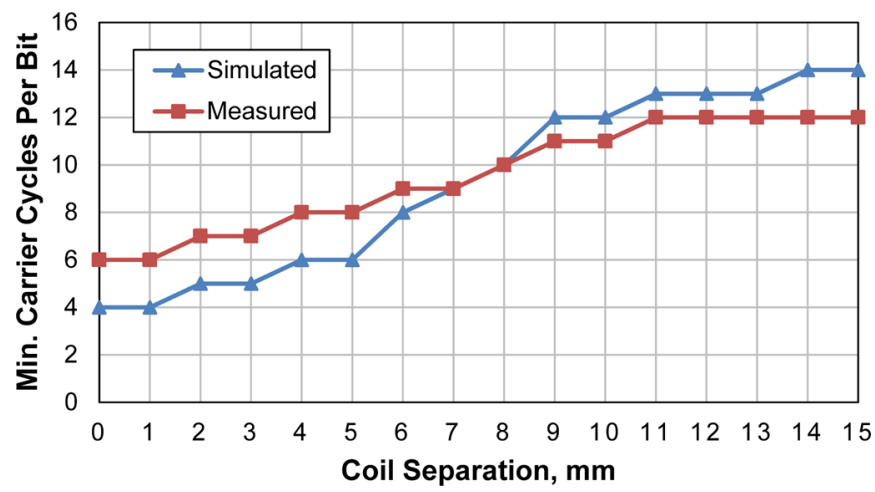

Fig. 19. Simulated and measured minimum number of carrier cycles needed to transmit one bit, for different coil separations.

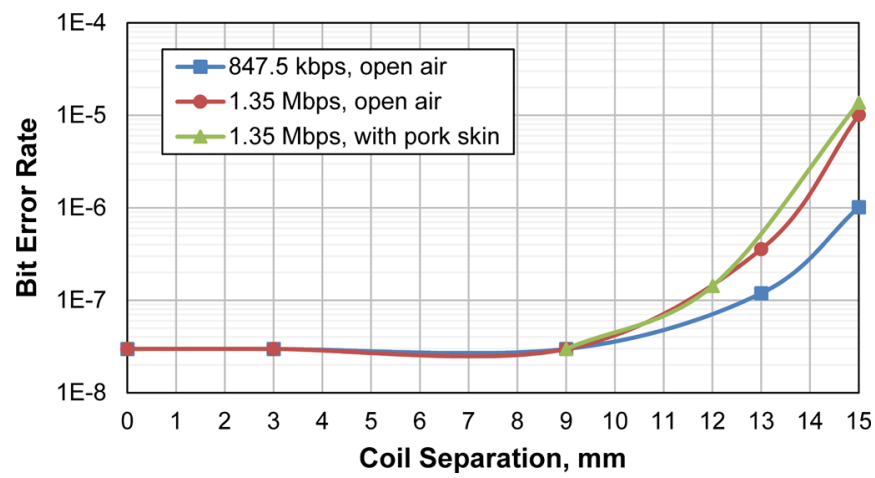

Fig. 20. Measured BER for data rates of $847.5 \mathrm{kbps}$ and $1.35 \mathrm{Mbps}$.

The minimum numbers of carrier cycles per bit were also recorded in these measurements and are plotted in Fig. 19 alongside the simulated values. It is shown that the chosen data rate of 16 carrier cycles per bit is enough for the specified working range of coil separation, but there is potential for a higher data rate, especially for small coil separations.

Bit error rate (BER) was measured with the PPSK modulator configured to continuously transmit a pseudorandom bit-stream generated from a 24-bit linear feedback shift register. For each measurement, 16.78 Mbits were collected from the PPSK demodulator and erroneous bits were counted to obtain the BER. The BER was measured with $R_{L}=1344 \Omega$, similar to the simulations. The measured BERs over a coil separation range between $0 \mathrm{~mm}$ and $15 \mathrm{~mm}$ at data rates of $847.5 \mathrm{kbps}$ and 1.35 Mbps in open air, are shown in Fig. 20. Over the working

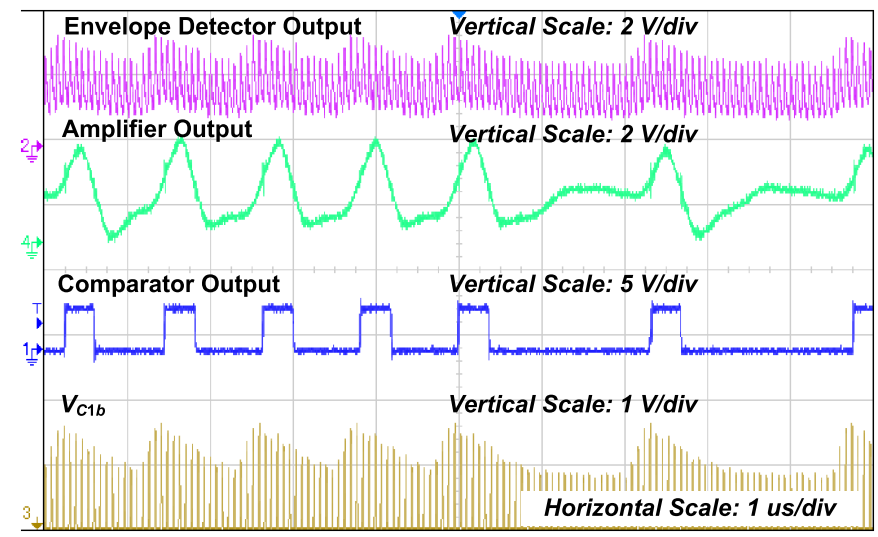

Fig. 21. Oscilloscope screenshot of signals in the PPSK demodulation stages.

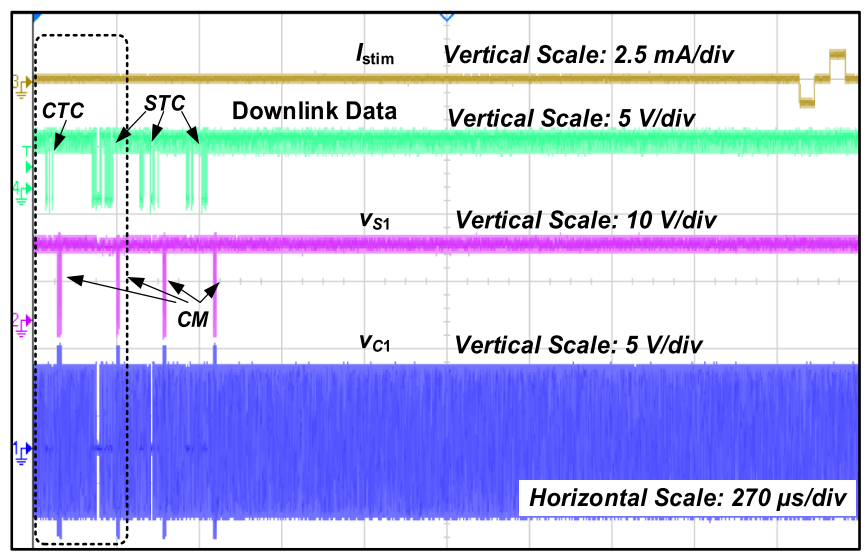

(a)

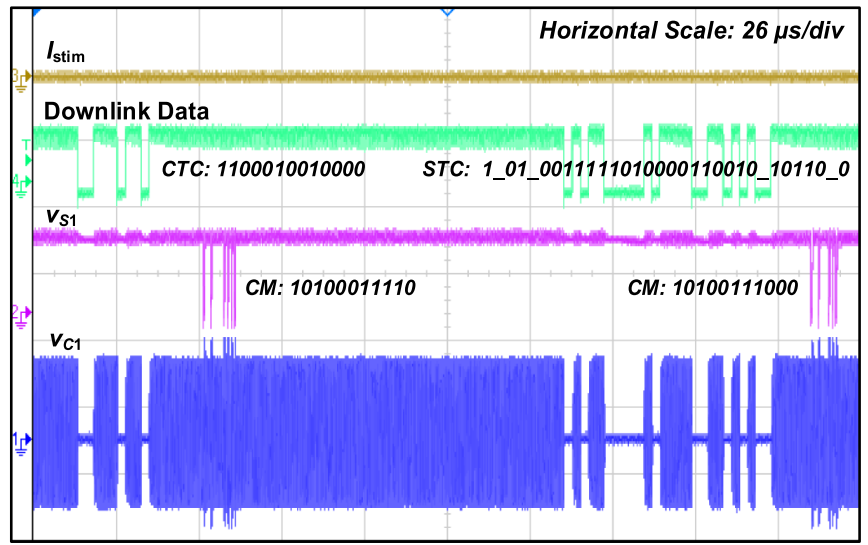

(b)

Fig. 22. Oscilloscope screenshot of telemetry operation for a handshake between the transmitter and the receiver.

range of $5 \mathrm{~mm}$ to $15 \mathrm{~mm}$ coil separation, the BER at $847.5 \mathrm{kbps}$ is below $1.01 \times 10^{-6}$ and the BER at $1.35 \mathrm{Mbps}$ is below $1 \times 10^{-5}$. To examine the effect of skin/tissue on the PPSK modulation the BER at $1.35 \mathrm{Mbps}$ was measured with a pork skin sample (of thickness 3-4 mm) attached to the secondary coil. The measured BER with the pork skin is also plotted in Fig. 20. The results show that the BER is only very slightly downgraded at long coil separations.

Fig. 21 shows the waveforms in the PPSK demodulation stages for extracting a 9-bit sequence (111110101). The captured waveforms are: the input to the envelope detector $\left(V_{C 1 b}\right)$, the 
TABLE III

COMPARISON With OTHER SingLE-INDUCTIVE-LinK DESIGNS

\begin{tabular}{|c|c|c|c|c|c|c|c|c|}
\hline \multirow[b]{2}{*}{ Reference } & \multirow{2}{*}{$\begin{array}{c}\text { Carrier } \\
\text { frequency } \\
(\mathrm{MHz})\end{array}$} & \multicolumn{2}{|c|}{ Downlink } & \multicolumn{3}{|c|}{ Uplink } & \multirow{2}{*}{$\begin{array}{c}\text { Coupling } \\
\text { coefficient in the } \\
\text { specified coil } \\
\text { separation }\end{array}$} & \multirow{2}{*}{$\begin{array}{c}\text { Power } \\
\text { delivered to the } \\
\text { load }(\mathrm{mW})\end{array}$} \\
\hline & & Modulation & $\begin{array}{c}\text { Data rate } \\
\text { (kbps) }\end{array}$ & Modulation & $\begin{array}{c}\text { Data rate } \\
(\mathrm{Mbps})\end{array}$ & BER & & \\
\hline [19] & 10 & BPSK & 1120 & LSK & n./a. & n./a. & 0.07 & $\geq 0.61$ \\
\hline [20] & 1 & - & - & LSK & 0.003 & $<1.6 \times 10^{-6}(\mathrm{PER})$ & $0.08-0.17$ & $\leq 250$ \\
\hline [21] & 13.56 & - & - & LSK & 0.5 & n./a. & $0.03^{*}$ & 20 \\
\hline [22] & 13.56 & BPSK & 1690 & LSK & 0.1 & n./a. & $0.04-0.12^{*}$ & 22.5 \\
\hline [23] & 13.56 & - & - & COOK & 6.78 & $<6 \times 10^{-7}$ & $\geq 0.34^{*}$ & $\leq 6.3$ \\
\hline This work & 13.56 & OOK & 400 & PPSK & 1.35 & $5.98 \times 10^{-8 * *}$ & $0.055-0.184$ & $\leq 100$ \\
\hline
\end{tabular}

*Estimated using equations (1)-(7) in [32] from the coil parameters given in the references. The coupling coefficients of [22] and [23] were estimated for both coaxial solenoid single-layer coil and planar spiral coil scenarios. In the latter the maximum spacing between two adjacent turns in the coil was set to $D_{\mathrm{MAX}} / N$, where $D_{\mathrm{MAX}}$ is the radius of the outermost turn, and $N$ is the total number of turns.

${ }^{* *} \mathrm{BER}$ at the critical coupling where the coil separation is $8 \mathrm{~mm}$. The BER over the full working range of coil separation is shown in Fig. 20.

output from the envelope detector, the output from the second amplifier, and the output digital bit-stream from the comparator. The probe positions capturing these waveforms are labeled in Fig. 11 as $A, B, C$, and $D$, respectively.

\section{Bi-Directional Communication}

Fig. 22 shows the recorded procedure of initiating biphasic stimulating pulses with the bi-directional communication over the inductive link as illustrated in Fig. 13. Fig. 22(a) shows four downlink data packets were sent to the implant using OOK, among which are one CTC packet for selecting the semicircular canal and three $S T C$ packets for setting the stimulation. The implant responds to each packet with a $C M$ packet using PPSK modulation. After the communication the stimulator starts generating biphasic current pulses $\left(I_{\text {stim }}\right)$, as shown in the top trace in Fig. 22(a). Fig. 22(b) shows a zoom-in view of the enclosed area in Fig. 22(a), which includes the CTC packet, the first STC packet and their corresponding $C M$ packets. The OOK and PPSK modulations are clearly visible in the trace of $v_{C 1}$.

\section{Comparison With State-of-the-Art}

A comparison of performance of the implemented power and data telemetry with other work is shown in Table III. This comparison is specific to designs that use a single inductive link for both power transfer and data communication, where the performance of the data link is limited by the implementation of the power link. PPSK modulation provides high load power and has a high data rate at low coupling with a good BER.

\section{CONCLUSION}

This paper has presented a telemetry system with simultaneous power delivery and bi-directional data transmission over a single pair of inductively coupled coils. The telemetry has been used to operate efficiently a vestibular prosthesis which requires both a high data rate and high power. An integrated PPSK modulator has been implemented in $0.6-\mu \mathrm{m}$ high voltage CMOS technology. A fast data rate of $1.35 \mathrm{Mbps}$ has been achieved for the uplink over the same pair of coils at a carrier frequency of $13.56 \mathrm{MHz}$ to transmit out neural recording data. The performance of the PPSK modulator and its dependence on the inductive link parameters have been investigated and verified.

\section{REFERENCES}

[1] F.-G. Zeng, S. Rebscher, W. Harrison, X. Sun, and H. Feng, "Cochlear implants: System design, integration, and evaluation," IEEE Rev. Biomed. Eng., vol. 1, pp. 115-142, 2008.

[2] G. J. Suaning and N. H. Lovell, "CMOS neurostimulation ASIC with 100 channels, scaleable output, and bidirectional radio-frequency telemetry," IEEE Trans. Biomed. Eng., vol. 48, no. 2, pp. 248-260, Feb. 2001.

[3] W. Liu, K. Vichienchom, M. Clements, S. C. DeMarco, C. Hughes, E. McGucken, M. S. Humayun, E. De Juan, J. D. Weiland, and R. Greenberg, "A neuro-stimulus chip with telemetry unit for retinal prosthetic device," IEEE J. Solid-State Circuits, vol. 35, no. 10, pp. 1487-1497, Oct. 2000.

[4] J. Coulombe, M. Sawan, and J.-F. Gervais, "A highly flexible system for microstimulation of the visual cortex: Design and implementation," IEEE Trans. Biomed. Circuits Syst., vol. 1, no. 4, pp. 258-269, Dec. 2007.

[5] M. Ortmanns, A. Rocke, M. Gehrke, and H.-J. Tiedtke, "A 232-channel epiretinal stimulator ASIC," IEEE J. Solid-State Circuits, vol. 42, no. 12, pp. 2946-2959, Dec. 2007.

[6] N. Donaldson, T. Perkins, I. Pachnis, A. Vanhoest, and A. Demosthenous, "Design of an implant for preventing incontinence after spinal cord injury," Artif. Organs, vol. 32, no. 8, pp. 586-591, Aug. 2008.

[7] X. Liu, A. Demosthenous, A. Vanhoestenberghe, D. Jiang, and N. Donaldson, "Active books: The design of an implantable stimulator that minimizes cable count using integrated circuits very close to electrodes," IEEE Trans. Biomed. Circuits Syst., vol. 6, no. 3, pp. 216-227, Jun. 2012.

[8] H.-M. Lee, H. Park, and M. Ghovanloo, "A power-efficient wireless system with adaptive supply control for deep brain stimulation," IEEE J. Solid-State Circuits, vol. 48, no. 9, pp. 2203-2216, Sep. 2013.

[9] K. Nie, L. Ling, S. M. Bierer, C. R. S. Kaneko, A. F. Fuchs, T. Oxford, J. T. Rubinstein, and J. O. Phillips, "An experimental vestibular neural prosthesis: Design and preliminary results with Rhesus monkeys stimulated with modulated pulses," IEEE Trans. Biomed. Eng., vol. 60, no. 6, pp. 1685-1692, Jun. 2013.

[10] N. S. Valentin, K. N. Hageman, C. Dai, C. C. Della Santina, and G. Y. Fridman, "Development of a multichannel vestibular prosthesis prototype by modification of a commercially available cochlear implant," IEEE Trans. Neural Syst. Rehabil. Eng., vol. 21, no. 5, pp. 830-839, Sep. 2013.

[11] D. Jiang, A. Demosthenous, T. Perkins, X. Liu, and N. Donaldson, "A stimulator ASIC featuring versatile management for vestibular prostheses," IEEE Trans. Biomed. Circuits Syst., vol. 5, no. 2, pp. 147-159, Apr. 2011.

[12] D. Jiang, D. Cirmirakis, and A. Demosthenous, "A vestibular prosthesis with highly-isolated parallel multichannel stimulation," IEEE Trans. Biomed. Circuits Syst., vol. 9, pp. 124-137, no. 1, Feb. 2015.

[13] C. A. Miller, P. J. Abbas, and C. J. Brown, "An improved method of reducing stimulus artifact in the electrically evoked whole-nerve potential," Ear Hear, vol. 21, no. 4, pp. 280-290, 2000.

[14] K. Nie, S. M. Bierer, L. Ling, T. Oxford, J. T. Rubinstein, and J. O. Phillips, "Characterization of the electrically evoked compound action potential of the vestibular nerve," Otol. Neurotol., vol. 32, no. 1, pp. 88-97, 2011. 
[15] R. R. Harrison, P. T. Watkins, R. J. Kier, R. O. Lovejoy, D. J. Black, B. Greger, and F. Solzbacher, "A low-power integrated circuit for a wireless 100-electrode neural recording system," IEEE J. Solid-State Circuits, vol. 42, no. 1, pp. 123-133, Jan. 2007.

[16] K. Abdelhalim, L. Kokarovtseva, J. L. Perez Velazquez, and R. Genov, "915-MHz FSK/OOK wireless neural recording SoC with 64 mixedsignal FIR filters," IEEE J. Solid-State Circuits, vol. 48, no. 10, pp. 2478-2493, Oct. 2013.

[17] M. Yin, D. A. Borton, J. Aceros, W. R. Patterson, and A. V. Nurmikko, "A 100-channel hermetically sealed implantable device for chronic wireless neurosensing applications," IEEE Trans. Biomed. Circuits Syst., vol. 7, no. 2, pp. 115-128, Apr. 2013.

[18] Z. Tang, B. Smith, J. H. Schild, and P. H. Peckham, "Data transmission from an implantable biotelemeter by load-shift keying using circuit configuration modulator," IEEE Trans. Biomed. Eng., vol. 42, no. 5, pp. 524-528, May 1995.

[19] Y. Hu and M. Sawan, "A fully integrated low-power BPSK demodulator for implantable medical devices," IEEE Trans. Circuits Syst. I, Reg. Papers, vol. 52, no. 12, pp. 2552-2562, Dec. 2005.

[20] G. Wang, W. Liu, M. Sivaprakasam, and G. A. Kendir, "Design and analysis of an adaptive transcutaneous power telemetry for biomedical implants," IEEE Trans. Circuits Syst. I, Reg. Papers, vol. 52, no. 10, pp. 2109-2117, Oct. 2005.

[21] H.-M. Lee and M. Ghovanloo, "An integrated power-efficient active rectifier with offset-controlled high speed comparators for inductively powered applications," IEEE Trans. Circuits Syst. I, Reg. Papers, vol. 58, no. 8, pp. 1749-1760, Aug. 2011

[22] S. Sonkusale and Z. Luo, "A complete data and power telemetry system utilizing BPSK and LSK signaling for biomedical implants," in Proc. 30th Annu. Int. Conf. IEEE Eng. Med. Biol. Soc., Vancouver, BC, Canda, Aug. 20-25, 2008, pp. 3216-3219.

[23] S. Ha, C. Kim, J. Park; S. Joshi, and G. Cauwenberghs, "Energy-recycling integrated 6.78-Mbps data $6.3-\mathrm{mW}$ power telemetry over a single 13.56-MHz inductive link," in Proc. Symp. VLSI Circuits, Dig Tech. Papers, Honolulu, HI, USA, 2014, pp. 1-2.

[24] S. Mandal and R. Sarpeshkar, "Power-efficient impedance-modulation wireless data links for biomedical implants," IEEE Trans. Biomed. Circuits Syst., vol. 2, no. 4, pp. 301-315, Dec. 2008.

[25] M. Kiani and M. Ghovanloo, "A 13.56-Mbps pulse delay modulation based transceiver for simultaneous near-field data and power transmission," IEEE Trans. Biomed. Circuits Syst., vol. 9, no. 1, pp. 1-11, Feb. 2015.

[26] M. Ghovanloo and S. Atluri, "A wide-band power-efficient inductive wireless link for implantable microelectronic devices using multiple carriers," IEEE Trans. Circuits Syst. I: Reg. Papers, vol. 54, no. 10, pp. 2211-2221, Oct. 2007.

[27] G. Simard, M. Sawan, and D. Massicotte, "High-speed OQPSK and efficient power transfer through inductive link for biomedical implants," IEEE Trans. Biomed. Circuits Syst., vol. 4, no. 3, pp. 192-200, Jun. 2010.

[28] D. Cirmirakis, D. Jiang, A. Demosthenous, N. Donaldson, and T. Perkins, "A fast passive phase shift keying modulator for inductively coupled implanted medical devices," in Proc. Eur. Solid-State Circuits Conf., Bordeaux, France, 2012, pp. 301-304.

[29] L. Zhou and N. Donaldson, "A fast passive data transmission method for ENG telemetry," Neuromodulation, vol. 6, no. 2, pp. 116-121, Apr. 2003

[30] L. Zhou, "Passive phase shift modulation for high-speed data transmission in implantable closed-loop neuroprostheses," Ph.D. dissertation, Dept. Med. Phys. Bioeng., University of London, London, U.K., 2005.

[31] D. Cirmirakis, A. Demosthenous, N. Saeidi, and N. Donaldson, "Humidity-to-frequency sensor in CMOS technology with wireless readout," IEEE Sensors J., vol. 13, no. 3, pp. 900-908, Mar. 2013.

[32] C. M. Zierhofer and E. S. Hochmair, "Geometric approach for coupling enhancement of magnetically coupled coils," IEEE Trans. Biomed. Eng., vol. 43, no. 7, pp. 708-714, Jul. 1996.

[33] K. Finkenzeller, RFID Handbook: Fundamentals and Applications in Contactless Smart Cards, Radio Frequency Identification and NearField Communication, 3rd ed. New York, NY, USA: Wiley-Blackwell, Jun. 18, 2010.

[34] R. J. Baker, CMOS: Circuit Design, Layout, and Simulation, 2nd ed. New York, NY, USA: Wiley-IEEE Press, 2008.

[35] [Online]. Available: http://www.cochlear.com/wps/wcm/connect/uk/ home/discover/cochlear-implants/nucleus-6-for-adults/nucleus-implantportfolio

[36] N. Donaldson and T. A. Perkins, "Analysis of resonant coupled coils in the design of radio frequency transcutaneous links," Med. Biol. Eng. Comput., vol. 21, no. 5, pp. 612-627, Sep. 1983.

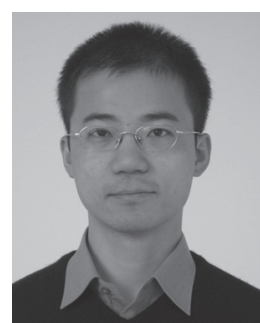

Dai Jiang (S'07-M'09) received the B.Sc. and M.Sc. degrees from Beijing University of Aeronautics and Astronautics, Beijing, China, in 1998 and 2001, respectively, and the Ph.D. degree from University College London (UCL), London, U.K., in 2009.

His Ph.D. work was on frequency synthesis. From 2001-2002, he was with Datang Telecom Group, China, working on developing FPGA functions for WCDMA signal processing. From 2006 to 2008, he was a Research Assistant, and since 2009, a Postdoctoral Research Associate, both with the Analog and Biomedical Electronics Group at UCL. His research interests include CMOS analog and mixed-signal integrated circuit design for biomedical applications.

Dr. Jiang is a member of the Biomedical Circuits and Systems Technical Committee of the IEEE Circuits and Systems Society.

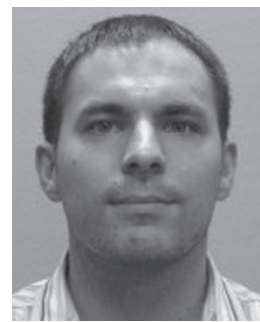

Dominik Cirmirakis received the B.Eng. degree in electrical and electronic engineering and the Ph.D. degree in biomedical electronics from University College London (UCL), London, U.K., in 2009 and 2013, respectively.

While at UCL, he was awarded the IEEE prize for the best communication project. His research interests include telemetry design for implantable devices, low-power analog and mixed signal integrated circuits for biomedical applications, in particular for cochlear and vestibular implants as well as firmware and software design for these devices.

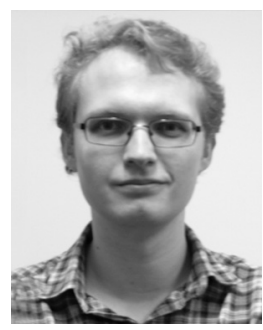

Matthew Schormans (S'15) was born in Basildon, U.K., in 1991. He received the M.Eng. degree in electronic and electrical engineering from University College London (UCL), London, U.K., in 2014.

Currently, he is working towards the Ph.D. degree in electronic and electrical engineering, with a focus on wireless power telemetry for implantable medical devices, at UCL. His research is concerned primarily with wireless power telemetry systems, but interests include inductive link modeling as well as general power converter and mixed signal design.

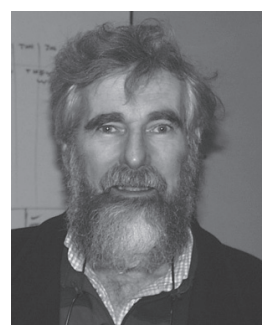

Timothy A. Perkins received the B.Sc. degree in physics from Bristol University, Bristol, U.K., and the M.Sc. degree in electrical engineering from London University, London, U.K., in 1971 and 1983, respectively.

In 1972, he designed stimulation controllers at the British Medical Research Council's Neurological Prostheses Unit under Prof. Giles Brindley. They jointly won the IEE prize for helping disabled people in 1985 , for their bladder control work. He joined Nick Donaldson's Implanted Devices Group at University College London, London, U.K., in 1992. His research interests continue to be in the design and practical use of stimulation controllers to rehabilitate the neurologically disabled. 


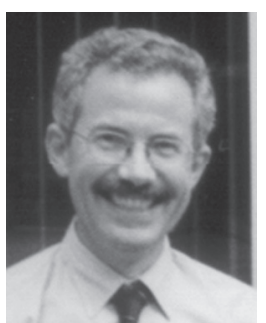

Nick Donaldson received the M.A. degree in engineering and electrical sciences from Cambridge University, Cambridge, U.K., and the Ph.D. degree from University College London (UCL), London, U.K., in 1976 and 1990, respectively.

From 1977 to 1992, he worked for the Medical Research Council, Neurological Prostheses Unit, London, U.K., under the direction of Prof. G. S. Brindley. In that period, his main field of research was the technology and use of implanted devices for the restoration of useful leg function to paraplegics. Since 1992, he has been with the UCL Department of Medical Physics and Bioengineering, where he is a Professor and leads the Implanted Devices Group. His research interests include the development of implanted devices that use natural nerve signals as inputs, especially for preventing incontinence, stimulators of nerve roots, the use of electrical stimulation for recreational exercise of paralyzed legs, and methods to encourage functional neurological recovery after injury.

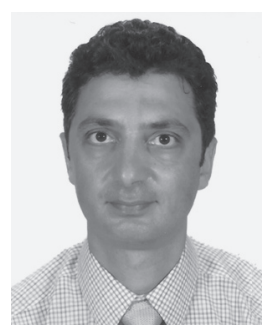

Andreas Demosthenous (S'94-M'99-SM'05) received the B.Eng. degree in electrical and electronic engineering from the University of Leicester, Leicester, U.K., the M.Sc. degree in telecommunications technology from Aston University, Birmingham, U.K., and the Ph.D. degree in electronic and electrical engineering from University College London (UCL), London, U.K., in 1992, 1994, and 1998, respectively.

Currently, he is a Professor in the UCL Department of Electronic and Electrical Engineering, where he leads the Analog and Biomedical Electronics Group. He has authored more than 250 articles in journals and international conference proceedings. His research interests include analog and mixed-signal integrated circuits for biomedical, sensor, and signal-processing applications.

Dr. Demosthenous is the Editor-in-Chief of the IEEE TRANSACTIONS ON Circuits AND Systems I: Regular Papers and an Associate Editor for the IEEE Trans actions on Biomedical CirCuits AND Systems. He is a member of the Technical Program Committee of several IEEE conferences including ESSCIRC and ISCAS. He is a Fellow of the Institution of Engineering and Technology and a Chartered Engineer. 